\title{
Edition of IGI 1
}

\begin{tabular}{|c|c|c|}
\hline & $\begin{array}{l}{[\ldots .] \mathrm{x}[\ldots]} \\
{[\ldots] \text { teqqi }}\end{array}$ & $\begin{array}{l}\mathrm{P}^{1}[\ldots] \mathrm{x}[\ldots] \\
2^{2}[\ldots] \text { you daub }[(\text { his eyes })] .\end{array}$ \\
\hline & NC i 1 ' & {$[\ldots$} \\
\hline & $\mathrm{NC}$ i 2 & {[} \\
\hline
\end{tabular}

\begin{tabular}{|c|c|c|}
\hline & $\begin{array}{l}\text { [... ana] pursiti tasahahat } \\
{[. . .] \text { inišsu teqqi }}\end{array}$ & $\begin{array}{l}{ }^{3}[\ldots] \text { you press (their saps) out [into] a } \\
\text { pursitu-bowl. }{ }^{4}[\ldots \text { (and)] you daub his } \\
\text { eyes. }\end{array}$ \\
\hline & \multicolumn{2}{|c|}{ 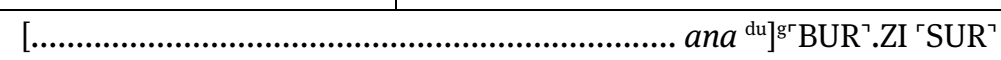 } \\
\hline & \multicolumn{2}{|c|}{ 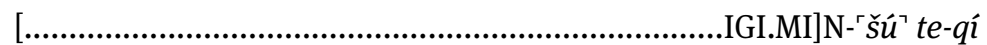 } \\
\hline
\end{tabular}

\begin{tabular}{|c|c|c|c|}
\hline $\begin{array}{l}5 \\
6\end{array}$ & \multicolumn{2}{|c|}{$\begin{array}{l}{[\ldots] \text {... }} \\
\text { lulâ rikibti arkabi lipi eșemti kurīti ša } \\
{[\text { immeri ... tasâk ...] }}\end{array}$} & $\begin{array}{l}5^{\prime}[. . .] \ldots{ }^{6}[\text { You pound }] \text { lulu } \hat{\text {-antimony, bat }} \\
\text { guano, marrow of a short bone of [ a } \\
\text { sheep ...] }\end{array}$ \\
\hline -g & NC i 5 ' & 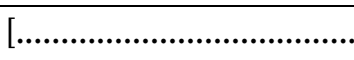 & 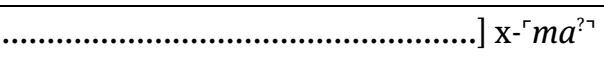 \\
\hline & NA i 1 ' & \multicolumn{2}{|c|}{$\begin{array}{l}\text { [KÙ.G]AN 'U }{ }_{5} \text {.ARGAB }{ }^{\text {mušen }} \text { İ’.UDU GİR.PAD.DA LÚGUD.DA ša [UDU ... } \\
\text { SÚD ...] }\end{array}$} \\
\hline & NC i $6{ }^{\prime}$ & 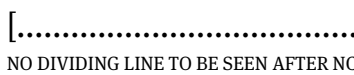 & ACE TOO BROKEN. \\
\hline
\end{tabular}

\begin{tabular}{|c|c|c|}
\hline 7 & $\begin{array}{l}\text { šīpa tasâk ina [takkussi ana libbi īnī̌su } \\
\text { tanappah] }\end{array}$ & $\begin{array}{l}\text { 7’You pound šìnu-paste, (which) [you blow } \\
\text { into his eyes] through a reed-[straw]. }\end{array}$ \\
\hline \multirow[t]{2}{*}{$7^{\prime}$} & \multicolumn{2}{|c|}{ 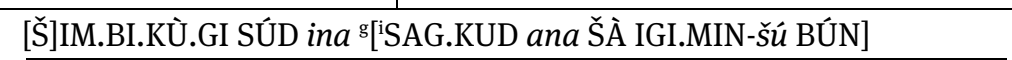 } \\
\hline & NC i 7 ' & 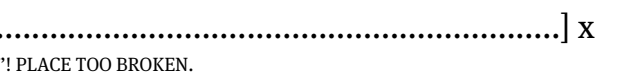 \\
\hline
\end{tabular}

Overview of IGI 1 Manuscripts and Parallels: AA=BAM 20; AB=BAM 159; AC=BAM 19; AD=BAM 22; AE=BAM 18; AmA=BAM 165; AX=KAL 7/12; bA=BM 41293+; EA=EMAR 6/4; HA=STT 279; NA=BAM 510; NB=BAM 513; NC=BAM 514; ND=BAM 515; NE=BAM 516; NJ=AMT 12/5; NQ=BAM 521; NU=AMT 14/3; sA=IRAQ 65; UA=AuOrS 23/27; uA=SpTU 50; UB=Ugaritica 5; UC=AuOrS 23/14; xA=CM 37

2 Open Access. (c) 2020 Markham J. Geller and Strahil V. Panayotov, published by De Gruyter. (c) BY-NC-ND This work is licensed under the Creative Commons Attribution-NonCommercial-NoDerivatives 4.0 License. https://doi.org/10.1515/9781501506550-004 


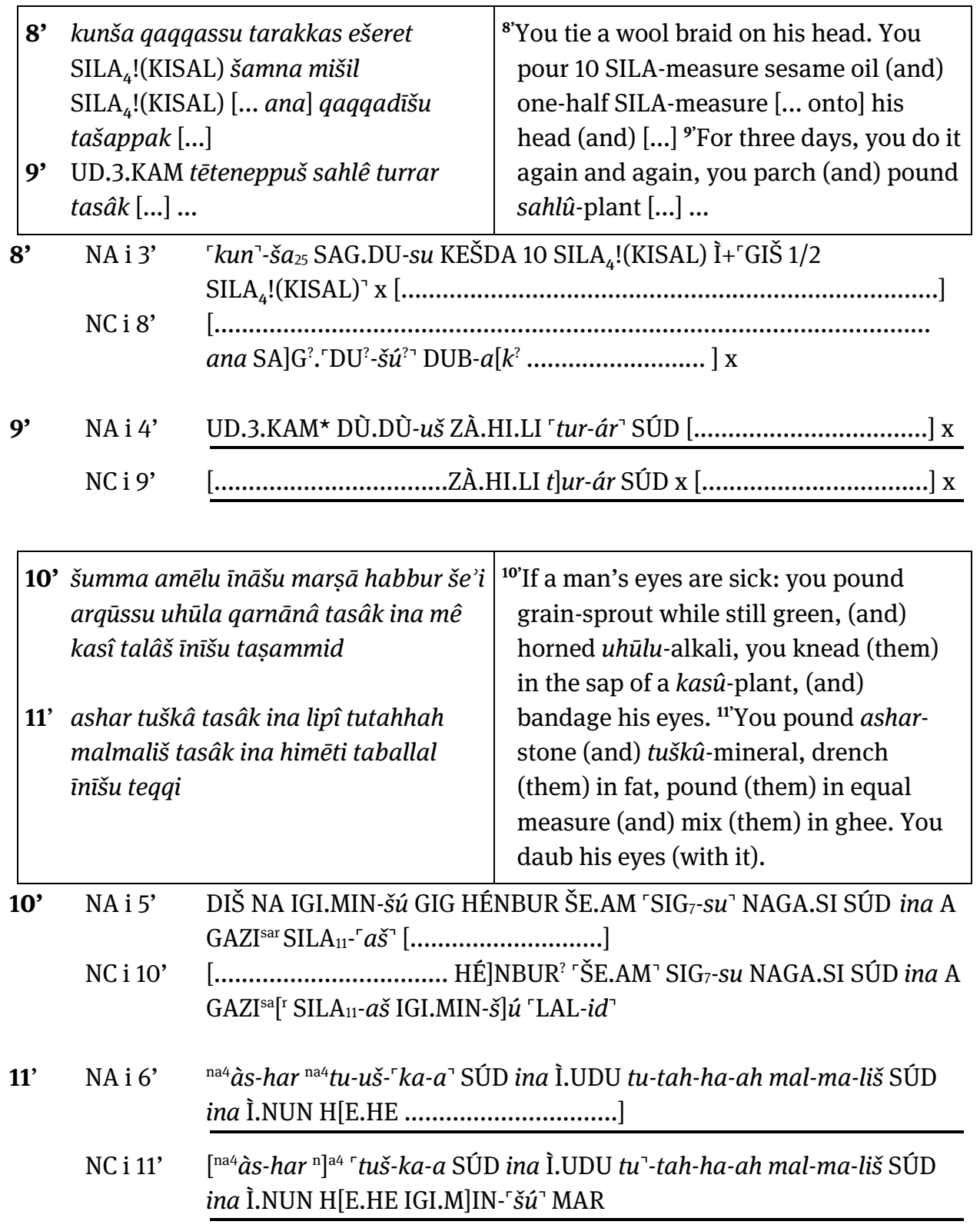

Overview of IGI 1 Manuscripts and Parallels: AA=BAM 20; AB=BAM 159; AC=BAM 19; AD=BAM 22; AE=BAM 18; AmA=BAM 165; AX=KAL 7/12; bA=BM 41293+; EA=EMAR 6/4; HA=STT 279; NA=BAM 510; NB=BAM 513; NC=BAM 514; ND=BAM 515; NE=BAM 516; NJ=AMT 12/5; NQ=BAM 521; NU=AMT 14/3; sA=IRAQ 65; UA=AuOrS 23/27; uA=SpTU 50; UB=Ugaritica 5; UC=AuOrS 23/14; xA=CM 37 
12' šumma amēlu ìnāšu marșā ešeret SILA $_{4}$ !(KISAL) šamna ana nakkapātī̌su tattanaddi šuhta ša aškāpi ina nādi tesêr

13’ ina nakkapātīšu tașammid hìl erî ashar šìna tasâk ina himēti taballal ìnī̌u tēteneqqīma iballut
12'If a man's eyes are sick: you keep on applying 10 SILA-measure of sesame oil on his temples. You spread copper patina from a leatherworker onto a waterproof leather. ${ }^{13}$ You bandage his temples (with the leather). You pound exudation of copper, ashar-stone (and) šìpu-paste, you mix (them) in ghee, you keep daubing his eyes, then he should recover.

12’ NA i 7’ 'DIŠ ${ }^{\prime}$ NA IGI.MIN-šú GIG 10 GA[ZI? ........ an]a 'SAG?.KI.MEŠ-šú ŠUB.MEŠ šu-uh-ta ša AŠGAB ina KUŠ.EDIN [te-ser]

NC i 12’ 'DIŠ NA? [IGI.MIN]-'šú GIG 10 SILA 4 !(KISAL)? İ.GIŠ ana SAG 'KI.MEŠšú ŠUB.MEŠ šu-uh-ta ša AŠ[GAB $t] e^{?-\ulcorner} \operatorname{ser}^{?\urcorner}$

13' NA i 8' [ina SA]G.KI.MEŠ-šú L[AL-id

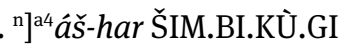

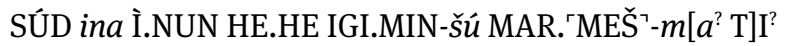

NC i 13’ rina S[AG.K]I?.[MEŠ-š]ú LAL-`id ILLU URUDU na4ás-har ŠIM.BI.KÙ.GI SÚD ina İ.NUN HE.HE [...........................................] $\mathrm{x}$

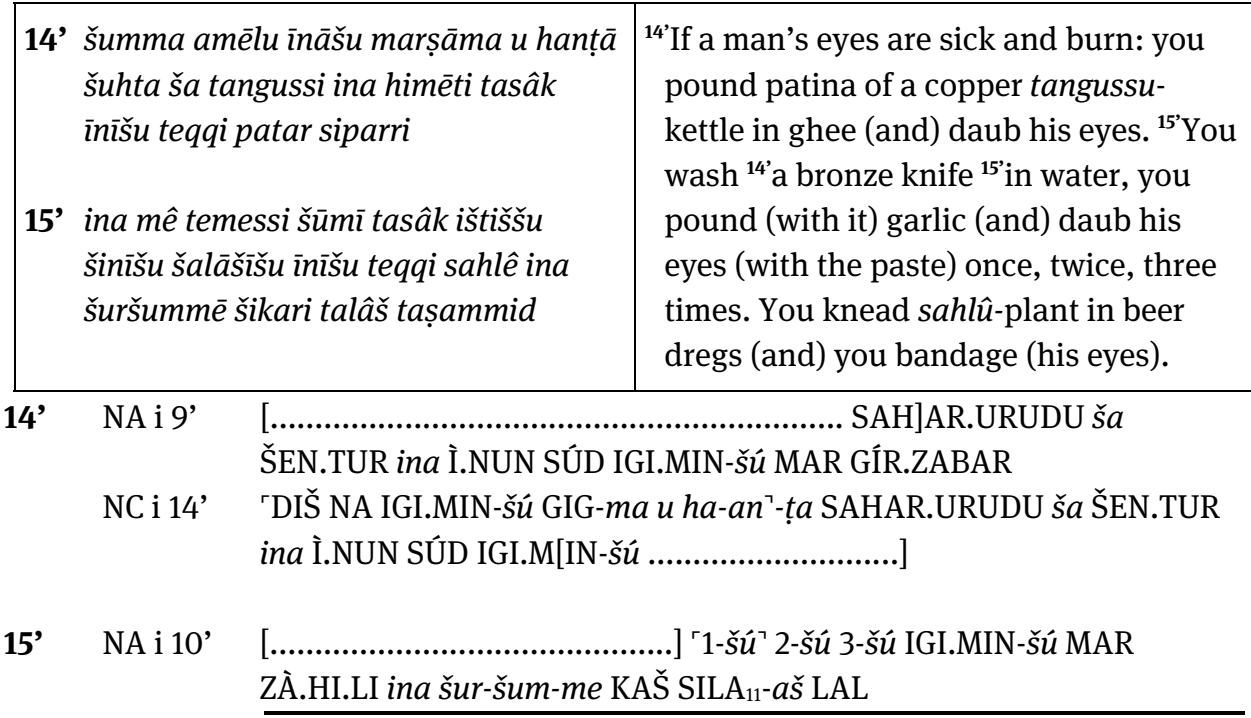

Overview of IGI 1 Manuscripts and Parallels: AA=BAM 20; AB=BAM 159; AC=BAM 19; AD=BAM 22; AE=BAM 18; AmA=BAM 165; AX=KAL 7/12; bA=BM 41293+; EA=EMAR 6/4; HA=STT 279; NA=BAM 510; NB=BAM 513; NC=BAM 514; ND=BAM 515; NE=BAM 516; NJ=AMT 12/5; $\mathbf{N Q = B A M ~ 5 2 1 ; ~ N U = A M T ~ 1 4 / 3 ; ~ S A = I R A Q ~ 6 5 ; ~ U A = A u O r S ~ 2 3 / 2 7 ; ~} \mathbf{u A}=$ SpTU 50; UB=Ugaritica 5; UC=AuOrS 23/14; $\mathbf{x A = C M ~} 37$ 


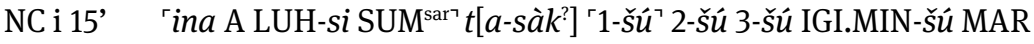
ZÀ.HI.LI in $[a$

\begin{tabular}{|c|c|c|c|}
\hline \multicolumn{3}{|c|}{$\begin{array}{l}\text { 16’ šumma ... [... šuhta] ša tangussi turrar } \\
\text { tahaššal ina himēti damiqte tasâk } \\
\text { 17’ enūma tēpušu [...] innī̌š teqqi }\end{array}$} & $\begin{array}{l}{ }^{16} \text { If ... [...] you parch (and) crush [patina] } \\
\text { from a copper tangussu-kettle, (and) you } \\
\text { pound (it) in a good quality ghee. } \\
{ }^{17} \text { When you have made it [... (and)] you } \\
\text { daub his eyes. }\end{array}$ \\
\hline & NA i 11 ' & \multicolumn{2}{|c|}{ 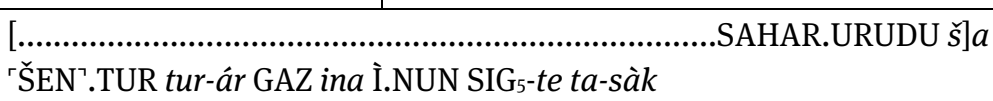 } \\
\hline & NB i 1 ' & \multicolumn{2}{|c|}{ [DIŠ ] x x x (x) [ } \\
\hline & NC i 16 ' & \multicolumn{2}{|c|}{ D[IŠ'? } \\
\hline & & \multicolumn{2}{|c|}{ 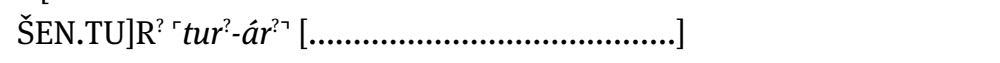 } \\
\hline \multirow[t]{2}{*}{$17^{\prime}$} & NA i 12 ' & \multicolumn{2}{|c|}{ [ } \\
\hline & NB i 2 ' & \multicolumn{2}{|c|}{ 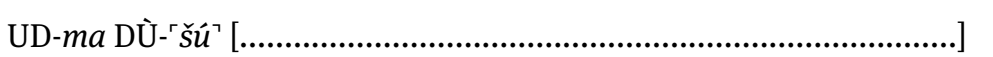 } \\
\hline
\end{tabular}

NC i 17' too broken

18’ aktam tasâk ina šikari tušabšal [...] ana libbi īnī̌s tunattak maštakal tasâk tașammid

19’ papparhâ tasâk tașammid sīha argāna barīrāta ina šizbi talâš tașammid

20’ lipi kalīti sāma ina șêti tanaddi ina itqūr abāri tasâk īnī̌su teqqi

${ }^{18}$ 'You pound aktam-plant (and) boil it in beer [...]. You drip (the mixture) into his eyes. You pound maštakal-plant and bandage (his eyes). ${ }^{19}$ You pound papparhû-purslane (and) bandage (his eyes). You knead sīhu-artemisia, argānuplant, (and) barīrātu-sagapenum in milk (and) you bandage (his eyes). ${ }^{20}$ You put reddish kidney fat in the sun-heat (and) pound (it) into a lead spoon(salve) (and) you daub his eyes.

18' NA i 13' an] $a$ 「ŠÀ? IGI.MIN-šú tu-na-ták ${ }^{\text {úN}} 6$.ÚŠ SÚD LAL

NB i 3' র ak-tam SÚD ina KAŠ ŠEG 6 X [.

NC i 18' too broken

Overview of IGI 1 Manuscripts and Parallels: AA=BAM 20; AB=BAM 159; AC=BAM 19; AD=BAM 22; AE=BAM 18; AmA=BAM 165; AX=KAL 7/12; bA=BM 41293+; EA=EMAR 6/4; HA=STT 279; NA=BAM 510; NB=BAM 513; NC=BAM 514; ND=BAM 515; NE=BAM 516; NJ=AMT 12/5; NQ=BAM 521; NU=AMT 14/3; sA=IRAQ 65; UA=AuOrS 23/27; uA=SpTU 50; UB=Ugaritica 5; UC=AuOrS 23/14; xA=CM 37 
19' NA i 14' ba-r]i-ra-ta ${ }_{5}$ ina GA ta-là-aš

LAL

NB i 4' BABBAR.HI ${ }^{\text {sar }}$ ta-sàk LAL ${ }^{\text {giš }}$ si-ha ${ }^{\text {giš }} a[r-g a-n u$..................................... $]$

NC i 19' too broken

20’ NA i 15 ’

i]na DÍLIM A.BÁR SÚD IGI.MINšú MAR

NB i 5’ İ.UDU 'ÉLLAG ${ }^{\top} \mathrm{SA}_{5}$ ina UD.DA ŠUB $\left.{ }^{\ulcorner}\right\urcorner-n\left[a^{?}\right.$ .............]

NC i 20’

...M]AR

21’ 'šmma amēlu īnāšu marșāma u katmā kikkirâna [...] supāla arqūssu

22' ina mê temessi tașammid inib errî šamma peșâ [tasâk ... īnī]šu teqqi
21'If a man's eyes are sick and closed: ${ }^{22}$ 'you wash kikkirânu-aromatic [..., and] ${ }^{21}$ 'supālu-juniper while still green in water, ${ }^{22}$ (and) you bandage (his eyes). You [pound] fruit of errû-colocynth, white plant [...], (and) you daub his [eyes].

21' NA i 16'

$\left.. \check{s}]^{i m r S ̌ E}{ }^{\urcorner} . L[I \ldots \ldots . . . s] u-{ }^{\ulcorner} p a-l a\right\urcorner \mathrm{SIG}_{7}-s u$

NB i 6,

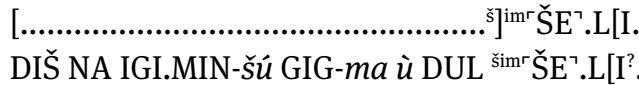

${ }^{\mathrm{S}} \mathrm{SIG}_{7}-\mathrm{Su} \mathrm{T}^{\mathrm{T}}$

22’ NA i 17' [ina A.M $] \mathrm{E}\left[\check{S}^{3}\right.$ ?

ÚKU]Š.LAGAB Ú.BABBAR x

$t] e-{ }^{\ulcorner} q \hat{\imath}^{?\urcorner}$

NB i 7’ ina A.MEŠ LUH-si LAL-id GURUN úÚKUŠ.LAGAB `Ú’ [.BABBAR SÚD .........................]

NC i 22’

Overview of IGI 1 Manuscripts and Parallels: AA=BAM 20; AB=BAM 159; AC=BAM 19; AD=BAM 22; AE=BAM 18; AmA=BAM 165; AX=KAL 7/12; bA=BM 41293+; EA=EMAR 6/4; HA=STT 279; NA=BAM 510; NB=BAM 513; NC=BAM 514; ND=BAM 515; NE=BAM 516; NJ=AMT 12/5; NQ=BAM 521; NU=AMT 14/3; SA=IRAQ 65; UA=AuOrS 23/27; uA=SpTU 50; UB=Ugaritica 5; UC=AuOrS 23/14; $\mathbf{x A = C M ~} 37$ 


\begin{tabular}{|c|c|c|}
\hline $24^{\prime}$ & $\begin{array}{l}\text { šumma amēlu ìnāšu marșāma ūmē } \\
\text { ma'dūti lā ipetti ina ummi [qaqqadīšu } \\
\text { ināššu șilla malâ] } \\
\text { qaqqassu tugallab ina ūme šalāšššu } \\
\text { līša tukașșa zēr [maštakal] turrar } \\
\text { ina himēti itqūr abāri tasâk īnīšu } \\
\text { teqqīma? ina'eš }\end{array}$ & $\begin{array}{l}{ }^{23} \text { 'If a man's eyes are sick and then he } \\
\text { cannot open (them) for many days, } \\
\text { while having ummu-fever [of his head } \\
\text { (and) his eyes are filled up with a film.] } \\
{ }^{24} \text { You shave his head (and) three times a } \\
\text { day, you cool off (his head) with dough. } \\
\text { You parch seeds [of maštakal-plant]. } \\
{ }^{25} \text { You pound (them) in the ghee of a lead } \\
\text { spoon(salve), (and) daub? his eyes, and } \\
\text { he should get better?. }\end{array}$ \\
\hline & 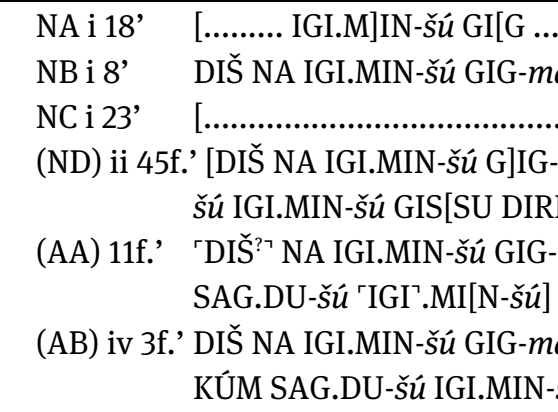 & 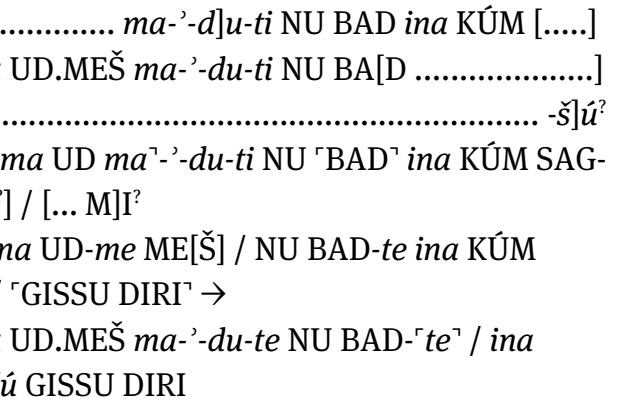 \\
\hline
\end{tabular}

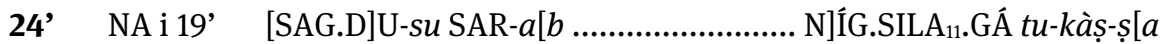

\begin{tabular}{|c|c|}
\hline NB i 8f.' & 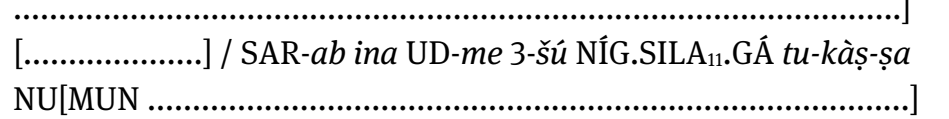 \\
\hline NC i 24f.' & 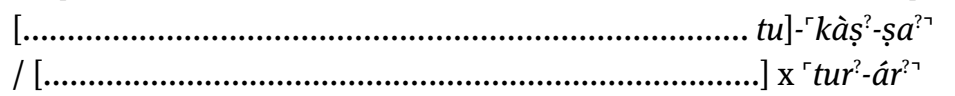 \\
\hline (ND) ii 46 & 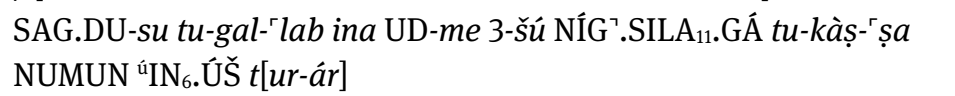 \\
\hline (AA) 13f.' & 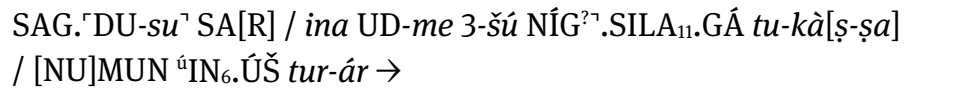 \\
\hline & SAG.DU-su SAR.SAR ina UD-me 3-šú ša NÍG. 'SILA11.GÁ? / tu-kàș- \\
\hline
\end{tabular}

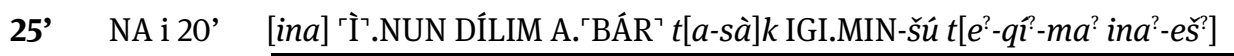

NB i 9f.' [...................] / DÍLIM A.BÁR ta-sàk IGI.MIN-šú [.............................]

\footnotetext{
Overview of IGI 1 Manuscripts and Parallels: AA=BAM 20; AB=BAM 159; AC=BAM 19; AD=BAM 22; AE=BAM 18; AmA=BAM 165; AX=KAL 7/12; bA=BM 41293+; EA=EMAR 6/4; HA=STT 279; NA=BAM 510; NB=BAM 513; NC=BAM 514; ND=BAM 515; NE=BAM 516; NJ=AMT 12/5; NQ=BAM 521; NU=AMT 14/3; sA=IRAQ 65; UA=AuOrS 23/27; uA=SpTU 50; UB=Ugaritica 5; UC=AuOrS 23/14; xA=CM 37
} 
NC i 26' in $] a^{?}-{ }^{r} e^{? ?}{ }^{?}$

THE DIVIDING LINE IS NOT VISIBLE!

(ND) ii 47 [ina İ.NUN DÍLIM] 'A A 'BÁR SÚD MAR A LUH-si GAZI ${ }^{\text {sar }}$ UGU 'IGI.MINšú GAR? EN? ú-za-ku'-ú GUR.G[UR]

(AA) 15ff.' ina İ.'NUN?? / [DÍLIM] 'A??.BÁR SÚD IGI.MIN-šú MAR.M[E(Š)] / [bul-ț $] u^{?}$ lat- $k[u$ ? $]$

(AB) iv 7f.' ina İ.NUN DÍLIM A.BÁR SÚD IGI.MIN-šú / [M]AR.MEŠ-ma bul-țu lat-ku

26’ šumma amēlu ìnāšu tābīla marșā šamaškilla uhašša ina šikari išatti šamna ana libbi īnišu tazarru[ma? ina'eš ]

\section{Alternative prescription 1}

27’ qēm aban suluppī turrar tasâk ina mê kasî talâš tukappat lām patān u'allat

\section{Alternative prescription 2}

28' muṣa'irāna arqa tașallip marassu ina himèti taballal īnī̌u teqqi

\section{Alternative prescription 3}

29’ hamšat SILA 4 !(KISAL) qēm hallūri šeššet SILA 4 !(KISAL) qēm kasî hamšat šiqil sahlê ina mê kasî talâš nakkaptāšu īnišu tașammid
26' If a man's eyes suffer from 'dryness': he (the patient) should chop šamaškilluonion (and) drink it in beer. (Then) you sprinkle? sesame oil into his eyes [and and he should get better?.]

\section{Alternative prescription 1}

27'You parch (and) pound powder of date stones, you knead (this flour) in the sap of a kasû-plant, you roll it (into a pill, which) he swallows before eating.

\section{Alternative prescription 2}

${ }^{28}$ 'You dissect a yellow-green mușa"irānufrog (and) you mix its bile in ghee. You daub his eyes (with it).

\section{Alternative prescription 3}

${ }^{29}$ You knead 5 SILA-measure chickpea powder, 6 SILA-measure powder of kasû-plant, (and) 5 shekels of sahluplant in the sap of a kasû-plant. You bandage his temple (and) his eyes.

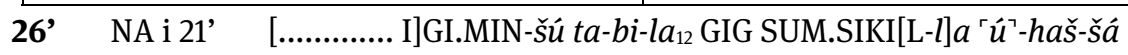
ina KAŠ NAG İ+GIŠ ana ŠÀ 'IGI.MIN-šú’ M[AR-ru-ma? ...................]

NB i 11’ DIŠ NA IGI.MIN-šú ta-bi-la 12 GIG SUM.SIKIL-'la? ú-haš-šá ina KAŠ NAG İ.GIŠ ana Š[À.

NC i $27^{\prime}$ .] $\mathrm{x}$

Overview of IGI 1 Manuscripts and Parallels: AA=BAM 20; AB=BAM 159; AC=BAM 19; AD=BAM 22; AE=BAM 18; AmA=BAM 165; AX=KAL 7/12; bA=BM 41293+; EA=EMAR 6/4; HA=STT 279; NA=BAM 510; NB=BAM 513; NC=BAM 514; ND=BAM 515; NE=BAM 516; NJ=AMT 12/5; NQ=BAM 521; NU=AMT 14/3; sA=IRAQ 65; UA=AuOrS 23/27; uA=SpTU 50; UB=Ugaritica 5; UC=AuOrS 23/14; $\mathbf{X A}=\mathrm{CM} 37$ 
27’ NA i 22’ [.... N]A $\mathrm{A}_{4}$ ZÚ.LUM.MA tur-âr SÚD ina A 'GAZI ${ }^{\text {sar }}$ ta?-la-aš tu-kap-pat la-a-am pa-'tan ú? ${ }^{?-}-a\left[l-l a t t^{?}\right]$

NB i 12’ Zİ NA $\quad$ 'ZÚ.LUM.MA tur-ár SÚD ina A GAZI ${ }^{\text {sar }}$ ta'-la-aš tu-kap-pat $l[a-a-a m$..................]

NC i 27’ too broken

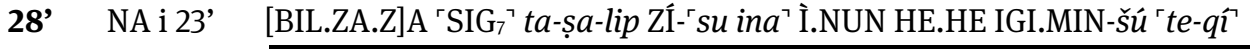

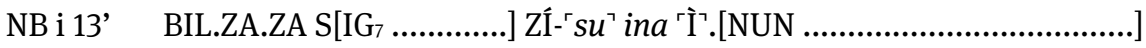

NC i 28, too broken

29' NA i 24'

$$
\text { [............ GÚ.G]AL } 6 \text { SILA } 4 \text { !(KISAL) Zİ GAZI }{ }^{\text {sar }} 5 \text { GÍN ZÀ.H[I.LI ina] `A }
$$
GAZI $^{\text {sar }}{ }^{\text {SILA }}{ }_{11}$-aš SAG.KI-šú IGI.MIN-šúu LAL

NB i 14’ 5 SILA $_{4}$ !(KISAL) Zİ .GÍ]N ZÀ.HI. 'LI' in[a A G]AZI ${ }^{\text {sar }}$ SI[LA ${ }_{11}-a \check{\text { š }}$ ..]

NC i 29' too broken

30’ šiptu [...] ... èrupū egûma

31’ [...] ... eli mê šalāš̌̌šu taqabbi
${ }^{30}$ 'Incantation ... they cloud over, they become lazy?. ${ }^{31}$ You say $[. .$.$] ... three$ times over water.

30' NA i 25' [ÉN x x x (x) ] x LAL A SAR NUMUN Ú x [ x ] x e-ru-pu e-gu-ma

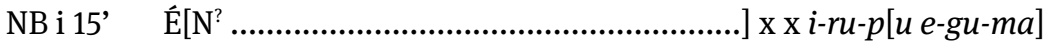

NC i 30' too broken

31' NA i 26’ [x x ] $\mathrm{x}^{\ulcorner} e^{\urcorner}-[l] i^{?}$ A 3-šú $\mathrm{DUG}_{4}$.GA

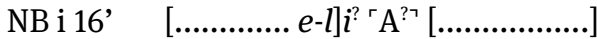

NC i 31’ too broken

32’ šumma amēlu [īnāšu marșā nīnû atā'išu kasû] sahlê māš $(t) u$ lipi kanakti zēr burāši
${ }^{32}$ If a man's [eyes] are [sick: nīnû-mint, atā'išu-plant, kasû-plant,] sahlû-plant, $m \bar{a} \check{s}(t) u$-twin plant, kanaktu-aromatic pith, seed of juniper, ${ }^{33}$ 'kammu-tanningfungus [of a leather worker. (These)

Overview of IGI 1 Manuscripts and Parallels: AA=BAM 20; AB=BAM 159; AC=BAM 19; AD=BAM 22; AE=BAM 18; AmA=BAM 165; AX=KAL 7/12; bA=BM 41293+; EA=EMAR 6/4; HA=STT 279; NA=BAM 510; NB=BAM 513; NC=BAM 514; ND=BAM 515; NE=BAM 516; NJ=AMT 12/5; NQ=BAM 521; NU=AMT 14/3; SA=IRAQ 65; UA=AuOrS 23/27; uA=SpTU 50; UB=Ugaritica 5; UC=AuOrS 23/14; $\mathbf{x A = C M ~} 37$ 


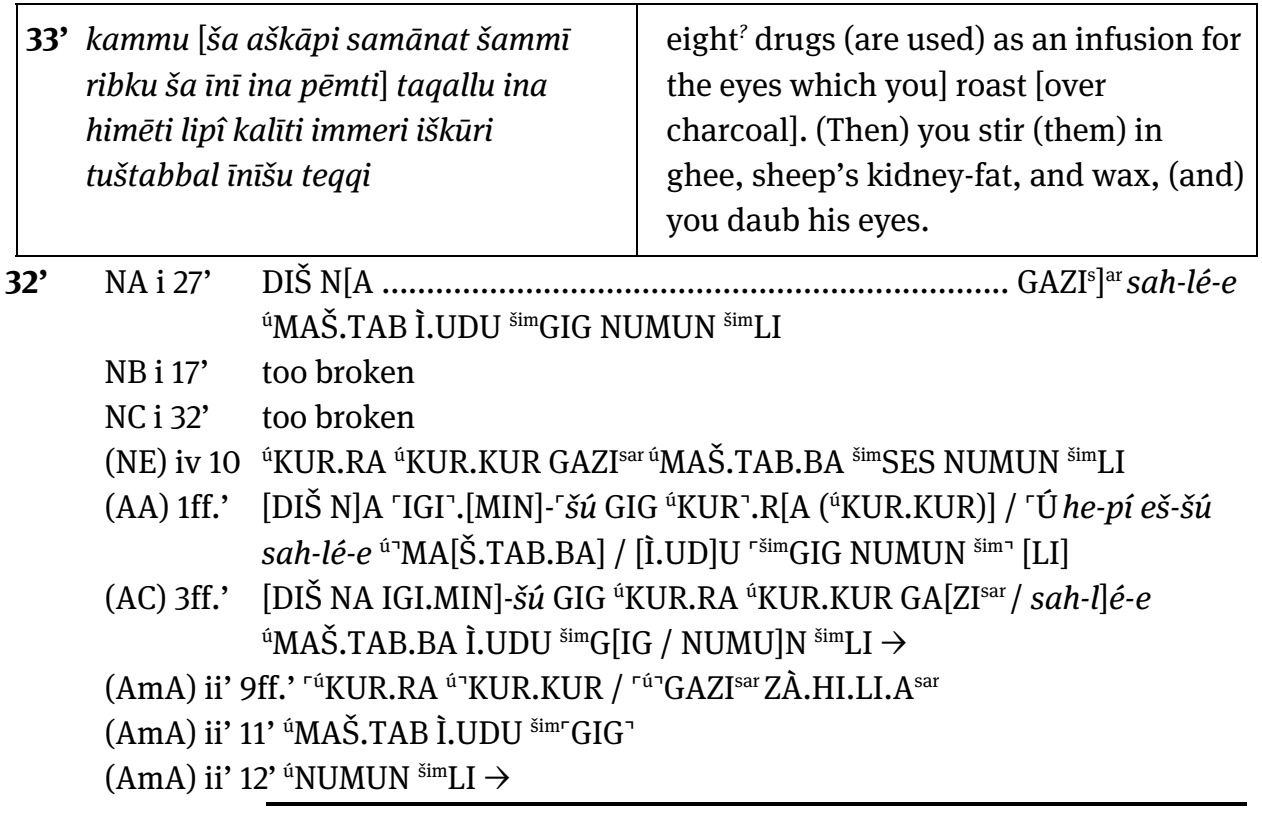

(sA) ii 15ff. DIŠ NA IGI.MIN-šú GIG.MEŠ 'KUR.RA úKUR.KUR / GAZI ${ }^{\text {sar }}$ sah-lé-e úMAŠ.TAB.BA İ.UDU šm GIG / NUMUN ${ }^{\text {šm } L I ~} \rightarrow$

33' NA i 28' kam-m[u ta-

qàl-l]u ina İ.NUN İ.UDU ÉLLAG UDU.NÍTA GAB.LÀL tuš-tab-bal IGI.MIN-šú MAR

NB i 18' too broken

NC i 33' too broken

(NE) iv 10 kam-mu šá AŠGAB 7 Ú.HI.A

(NE) iv 11 rib-ku ša IGI.MIN ina NE ta-qal-lu ina İ.UDU GAB.LÀL $u$ İ.NUN SÚD IGI.MIN-šú MAR

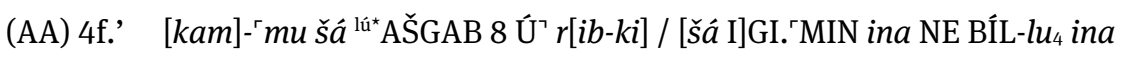
Ì?.[UDU]

(AA) 6’ [ÉLL]AG `UDU.NÍTA GAB.LÀL u İ.NUN? SÚ[D MAR]

(AC) 5f.' kám-mu šá lúAŠGAB 8 Ú rib- ${ }^{\ulcorner} k \imath^{\urcorner} /$[šá I]GI.MIN.MEŠ ina NE BÍL-lu $u$ İ.UDU ÉLLAG UD[U NÍ]TA

(AC) 6’ [GAB.LÀ]L $u$ İ.NUN SÚD IGI.MIN-šú 'MAR`

Overview of IGI 1 Manuscripts and Parallels: AA=BAM 20; AB=BAM 159; AC=BAM 19; AD=BAM 22; AE=BAM 18; AmA=BAM 165; AX=KAL 7/12; bA=BM 41293+; EA=EMAR 6/4; HA=STT 279; NA=BAM 510; NB=BAM 513; NC=BAM 514; ND=BAM 515; NE=BAM 516; NJ=AMT 12/5; NQ=BAM 521; NU=AMT 14/3; SA=IRAQ 65; UA=AuOrS 23/27; uA=SpTU 50; UB=Ugaritica 5; UC=AuOrS 23/14; xA=CM 37 
(AmA) ii’ 12’ kám-ma šá rlú $\mathrm{AŠGAB}^{\urcorner}$

(AmA) ii’ 13’ 8 Ú rib-ku ša IGI.MIN 'BIL?

(AmA) ii’ 14' ina İ.UDU GAB.LÀL İ.NUN.NA HE.HE

(sA) ii 17f. KÙ.GAN 8? Ú.HI.A / rib-ki DÙG.GA šá IGI.MIN ta-qal-lu ina İ.NUN.NA

(sA) ii 19 İ.UDU ÉLLAG GAB.LÀL SÚD IGI.MIN-šú MAR

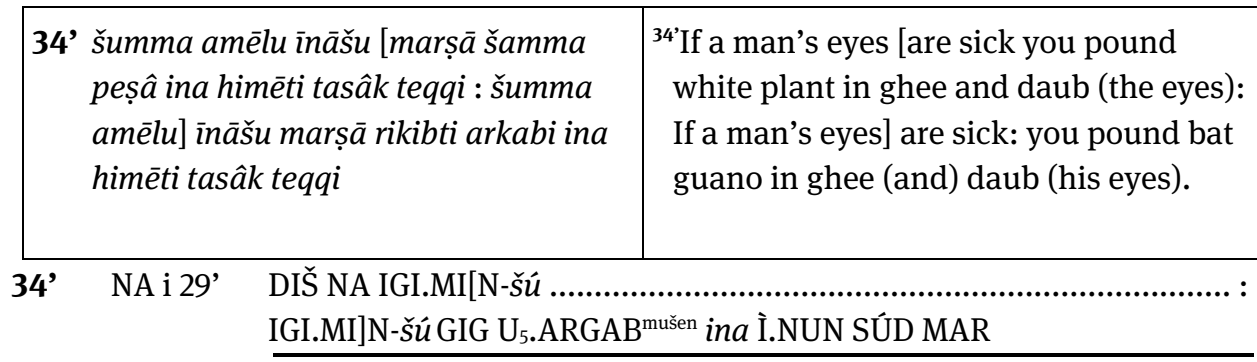

NB i 19' too broken

NC i 34' too broken

(AC) 11f.' [DIŠ N]A 'IGI.MIN-šú GIG Ú.BABBAR ina İ.NUN SÚD MAR / [DIŠ? KIMIN?] ' $\mathrm{U}_{5} . \mathrm{ARGAB}^{\mathrm{mušen}}$ ina İ.NUN SÚD MAR

35’ šumma amēlu ìnāšu [marșā aban gabî ina himēti tasâk teqqi : šumma amēlu] innāšu marșā sahlê ina šizbi tušabšal tașammid
${ }^{35}$ 'If a man's eyes [are sick: You pound alum in ghee (and) daub (his eyes): If a man's] eyes are sick you boil sahlû-plant in milk (and) bandage (his eyes with it).

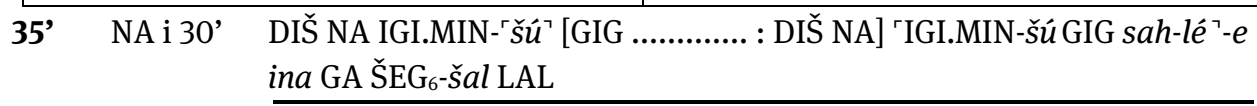

NB i 20' too broken

NC i 35’ 'DIŠ NA IGI.MIN-šú? [

(NQ) 8, [DIŠ NA IGI.MIN]-šú GIG sah-lé- ${ }^{\top} e^{\urcorner}[$.

(AC) 13’ [DIŠ KIMIN N]A ${ }_{4}^{\prime}$ 'ga-bi-i ina İ’.NUN SÚD MAR

(AC) 14’ [DIŠ? KIMIN? sa]h-'lé'-e ina GA SÚD MAR

Overview of IGI 1 Manuscripts and Parallels: AA=BAM 20; AB=BAM 159; AC=BAM 19; AD=BAM 22; AE=BAM 18; AmA=BAM 165; AX=KAL 7/12; bA=BM 41293+; EA=EMAR 6/4; HA=STT 279; NA=BAM 510; NB=BAM 513; NC=BAM 514; ND=BAM 515; NE=BAM 516; NJ=AMT 12/5; NQ=BAM 521; NU=AMT 14/3; sA=IRAQ 65; UA=AuOrS 23/27; uA=SpTU 50; UB=Ugaritica 5; UC=AuOrS 23/14; xA=CM 37 
36’ šumma amēlu ìnāšu dāma [malâma urra u mūša lā iṣallalma ...] ... šumma libbi īnišu sām

37’ 'ināšu katmā kasâ turrar [...] kabūt immeri ina šizbi musukkati talâš tașammid

38' ina šêri tapațțaršuma ashar ina himēti tasâk īnišu teqqi [(...)] šuruš šammi rapādi ina patar siparri tanakkis

39’ țurri nabāsi u šīpāti peșâti tațammi talammi tarakkas nakkapātī̌u kilaltān tarakkasma? ... tušeșșēma ìnī̌u [teqqi]
${ }^{36}$ 'If a man's eyes are [full of] blood [and he cannot sleep day and night ....], if the inner part of his eyes is red, ${ }^{37}$ '(and) his eyes are closed: you parch kasû-plant [...], you knead sheep dung in the milk from a woman in maternity (and) bandage (his eyes). ${ }^{38}$ In the morning you loosen it (the bandage), and then you pound ashar-stone in ghee, (and) daub his eyes $[(. .)$.$] . You cut with a$ bronze knife the root of rapādu-diseaseplant. ${ }^{39}$ You spin a cord of red and white wool, you wrap and bind up (the root of rapādu-plant with the wool, and) tie (it) on his both temples, and ... you remove (it), and then [daub] his eyes.

36' NA i 31' DIŠ NA IGI.MIN-šú MÚ[D ] $\mathrm{RI}$ šum ${ }_{4}-m a$ ŠÀ IGI.MIN-šú SA

NB i 21' $[$.

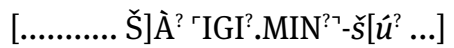

NC i 36f.' ' 'DIŠ NA IGI.MIN-šú MÚD DIRI-ma ur-ra u GE la $^{\urcorner\urcorner-s ̦ a[l-l a l-m a ~}$ / 'řum 4 -ma ŠÀ IGI.MIN-šú $\mathrm{SA}_{5}{ }^{\urcorner} \rightarrow$

(xA) 8f.' 'DIŠ NA IGI'.M[IN-šú MÚD] 'DIRI.MEŠ-ma ur-ri u GE 6 la i-ṣa-lal-ma

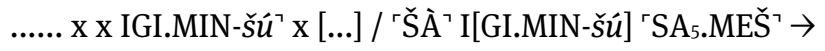

37’ NA i 32’ IGI.MIN-šú DUL-m[a munus] 'Ú'.ZÚG SILA ${ }_{11}$-aš LAL-id

NB i 22’ $[\ldots$ ŠU]RUN 'UDU' ina GA munus Ú.ZÚ[G ..]

NC i 37f.' 'IGI.MIN-šúú DUL-ma GAZI ${ }^{\mathrm{sar}}$ tur-ár KI $\mathrm{K}$ x [. ...] / 'SILA ${ }_{11}-a \check{\text { LAL-id }} \rightarrow$

(xA) 9f.' ' 'IGI.MIN-šú DUL-ma GAZI ${ }^{\mathrm{sar}}$ tur-ár ${ }^{\urcorner}$ / SILA $_{11}-a \check{s}^{\ulcorner} \mathrm{LAL}^{\urcorner} \rightarrow$

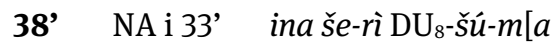
ina GÍR ZAB]AR KUD-[iṣ?]

Overview of IGI 1 Manuscripts and Parallels: AA=BAM 20; AB=BAM 159; AC=BAM 19; AD=BAM 22; AE=BAM 18; AmA=BAM 165; AX=KAL 7/12; bA=BM 41293+; EA=EMAR 6/4; HA=STT 279; NA=BAM 510; NB=BAM 513; NC=BAM 514; ND=BAM 515; NE=BAM 516; NJ=AMT 12/5; NQ=BAM 521; NU=AMT 14/3; sA=IRAQ 65; UA=AuOrS 23/27; uA=SpTU 50; UB=Ugaritica 5; UC=AuOrS 23/14; xA=CM 37 
NB i 23’

SUHU]Š? ' 'U ra-pa-di ina GÍR ZA[BAR

NC i 38’ 'ina še-rì DU 8 -šú-ma ${ }^{\text {na4}}$ às-har ina İ.NUN SÚD IGI.MIN-šú ${ }^{7}$ MA[R

(xA) 10f.' [ina še-r]ì 'DU8-šú-ma <ana> TIN-šú na4? as-har ina İ.NUN SÚD IGI.MINšú MAR SUHUŠ` Ú $r a^{?}-p a^{?}-d i^{?\urcorner} /$ rina GÍR? ZABAR$^{?} \mathrm{KUD}^{?\urcorner} \rightarrow$

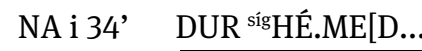

NB i 24f.' SÍ]G 'BABBAR NIGIN'-m[i? tar $] a^{\ulcorner} k a ̀ s^{\urcorner}$ SAG.KI.MEŠ-šú ki-lál-t[an? ... / ...] È-`ma? IGI.MIN-šú t[e?-(eq)-qí]

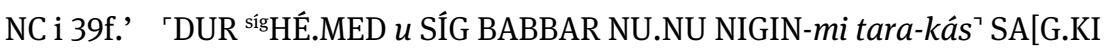
..] / 'tara? -kás?-ma? x x È-ma? I[GI.MIN-šú ........]

(xA) 11f.' 'rsíg HÉ?.[MED.D]A SÍG BABBAR NU.NU NIGIN-mi tara-kás SAG.KI.MINšú ki-lat-t[an] / 'tara-kás-m[a] x? È-ma IGI.MIN-šú x

\begin{tabular}{|c|c|}
\hline $\begin{array}{l}\text { 40’ šumma amēlu ìnāšu dāma šunnu'ā zēr } \\
\text { egemgīri dišip šadê lèra taballal [tasâk } \\
\text { (innišu) teqqi] }\end{array}$ & $\begin{array}{l}4^{40} \text { If a man's eyes are suffused with blood: } \\
\text { you mix seeds of egemgiru-plant, } \\
\text { mountain honey, (and) lēru-paste, [you } \\
\text { pound (them) and daub (his eyes)]. }\end{array}$ \\
\hline $\begin{array}{l}\text { Alternative prescription } 1 \\
\text { 41’ ištēn šiqil rikibti arkabi mišil } \\
\quad \text { SILA }_{4} \text { !(KISAL) šamma peșâ šušši } \\
\quad \text { emesalli ina [himēti tasâk īnišš teqqi] }\end{array}$ & $\begin{array}{l}\text { Alternative prescription } 1 \\
\text { 41' }^{1} \text { You pound] one shekel of bat guano, } \\
\text { half SILA-measure of white plant (and) } \\
\text { one-sixth (SILA-measure) emesallu- } \\
\text { saline solution in [ghee and daub his } \\
\text { eyes with (it)]. }\end{array}$ \\
\hline $\begin{array}{l}\text { Alternative prescription } 2 \\
\text { 42’ 'šamma peṣâ tābīla ana libbi ìnīšu } \\
\text { tazarru dāmāta ina himēti [...] } \\
\text { 43’ zê șurāri ina lipî eșemti kurīti tasâk lāa } \\
\text { patān [...] }\end{array}$ & $\begin{array}{l}\text { Alternative prescription } 2 \\
\text { 42’You spatter white plant in a dry state } \\
\text { into his eyes, [you pound] dāmātu-paste } \\
\text { in ghee [and .... }{ }^{43^{3}} \text { You pound lizard's } \\
\text { excrement in the marrow of a short } \\
\text { bone, without (him) eating, [...]. }\end{array}$ \\
\hline
\end{tabular}

Overview of IGI 1 Manuscripts and Parallels: AA=BAM 20; AB=BAM 159; AC=BAM 19; AD=BAM 22; AE=BAM 18; AmA=BAM 165; AX=KAL 7/12; bA=BM 41293+; EA=EMAR 6/4; HA=STT 279; NA=BAM 510; NB=BAM 513; NC=BAM 514; ND=BAM 515; NE=BAM 516; NJ=AMT 12/5; NQ=BAM 521; NU=AMT 14/3; sA=IRAQ 65; UA=AuOrS 23/27; uA=SpTU 50; UB=Ugaritica 5; UC=AuOrS 23/14; xA=CM 37 


\begin{tabular}{|c|c|}
\hline $\begin{array}{l}\text { Alternative prescription } 3 \\
\text { 44’ hamiš utțat šamma peșâ ina šamni } \\
\text { tasâk īnāšu teqqi rikibti arkabi ina } \\
\quad \text { himēti tasâk innāšu? teqqi? }\end{array}$ & $\begin{array}{l}\text { Alternative prescription } 3 \\
{ }^{44^{\prime}} \text { You pound five grain measure of white } \\
\text { plant in sesame oil, you daub his eyes. } \\
\text { You pound bat guano in ghee, (and) you } \\
\text { daub his eyes (with it). }\end{array}$ \\
\hline
\end{tabular}

40’ NA i 35’ DIŠ NA IGI.MIN-šú MÚD šu-u[n-nu-'a

NB i 26'

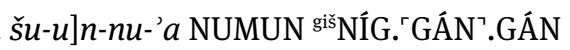
LÀL.KUR.RA SAHAR.KÙ.GI HE.H[E ............................................]

NC i 41' 'DIŠ NA IGI.MIN-šú MÚD šu-un-nu-'a NUMUN gišNÍG.GÁN.GÁN LÀL?.$K U[R . R A$

(xA) 14’ DIŠ N[A šu-u]n-n[u-'a gišNíG.GÂ]N.GÁN ${ }^{\text {sar!(di) }}$ LÀL.KUR.'RA SAHAR.KÙ'.GI 1-niš HE.HE S[ÚD (IGI.MIN-šú) MAR]

41 $\quad$ NA i 36’ 1 GÍN U . ARGAB $^{\text {mušen }} 1 /[2$

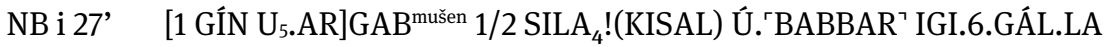
${ }^{\text {mun }}$ eme-sal- 'li’ $i\left[\mathrm{na}^{\text {? }}\right.$

NC i 42’ ' '1 GÍN U5.ARGAB ${ }^{\text {mušen }} 1 / 2$ GÍN Ú.BABBAR IGI.6.GÁL.LA ${ }^{\text {MUN? }} e m[e$-sal-lì

(AB) iv 25f.' 1 GÍN U 5.ARGAB ${ }^{\text {mušen }}$ 1/2 G[ÍN/GA]ZI 'Ú.BABBAR' / 15 ŠE mun eme-sal-lì ina İ.NUN SÚD 'IGI.MIN-šú MAR ${ }^{\prime}$

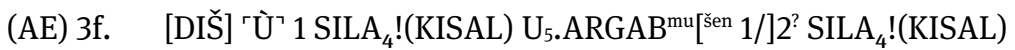

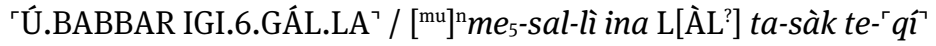

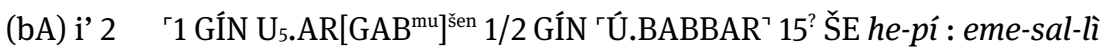
ina İ.NUN SÚD te-qí

42’ NA i 37’ Ú.BABBAR ta-bi-la ${ }_{12}$ ana ŠÀ IGI.MI[N-šú

NB i 28’ [Ú.BABBAR $t a-b] i^{?}-l a_{12}{ }^{2}$ ana ŠÀ IGI.MIN-šú ${ }^{7}$ MAR-ru ŠIM.BI.SIG 7. SIG $_{7}$ ina 'Ì? [.NUN ...]

NC i 43’ 'Ú.BABBAR ta-bi-la $a_{12}$ ana ŠÀ IGI.MIN-šú MAR-ru ŠIM.BI'.SI[G $\left[G_{7} . S_{G} G_{7}\right.$ .......................]

Overview of IGI 1 Manuscripts and Parallels: AA=BAM 20; AB=BAM 159; AC=BAM 19; AD=BAM 22; AE=BAM 18; AmA=BAM 165; AX=KAL 7/12; bA=BM 41293+; EA=EMAR 6/4; HA=STT 279; NA=BAM 510; NB=BAM 513; NC=BAM 514; ND=BAM 515; NE=BAM 516; NJ=AMT 12/5; NQ=BAM 521; NU=AMT 14/3; SA=IRAQ 65; UA=AuOrS 23/27; uA=SpTU 50; UB=Ugaritica 5; UC=AuOrS 23/14; xA=CM 37 
43’ NA i 38’ ŠE 10 $_{10}$ EME.ŠID ina İ.UDU GÌR.PAD.DU LÚGUD.DA [. ....]

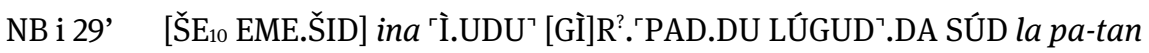
[...]

NC i 44’ ' 'ŠE 10 EME.ŠID ina İ.UDU GÌR.PAD.DU LÚGUD.DA SÚD? $l[a$ ...]

44’ NA i 39’ 5 ŠE Ú.BABBAR ina İ.GIŠ SÚD IGI.MIN-šú MA[R

NB i 30’ S]ÚD IG[I.MIN-šú] 'MAR ${ }^{\urcorner}[U]_{5 .}{ }^{r}$ ARGAB ${ }^{\text {mušen }}$ ina İ.NUN S[ÚD ..]

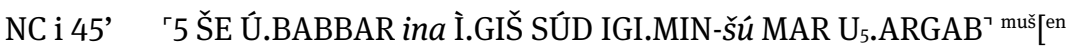
IGI.MIN]-`šú? MAR ?

45’ šumma amēlu īnāšu dāma malâ dāmāta ina himēti tasâk teqqi

\section{Alternative prescription 1}

46' maštakal tarmuš malmališ taballal ina himēti nikipta [zikar u sinniš] tasâk innišu teqqi

\section{Alternative prescription 2}

47' aban tašrîti ina ru'ti tasâk teqqi : šimbizidâ? (...) ina šizbi musukkati [tasâk ...] hašhūra tasâk teqqi

\section{Alternative prescription 3}

48’ dāma ša libbi šahî ana libbi īnī̌su [tunattak ...] ... ìnī̌u tașammid
45'If a man's eyes are full of blood: you pound dāmātu-paste in ghee, you daub (his eyes).

\section{Alternative prescription 1}

${ }^{46}$ You mix in equal measure maštakalplant and tarmuš-lupine, you pound [male and female] nikiptu-plant in ghee, (and) daub his eyes.

\section{Alternative prescription 2}

${ }^{47}$ You pound tašrītu-stone in spittle (and) daub (his eyes with it) : [You pound] šimbizid $\hat{u}$-galena? (...) in the milk from a woman in maternity, you pound ... (and) an apple, (and) daub (his eyes).

\section{Alternative prescription 3}

${ }^{48}$ You [drip] into his eyes blood from a pig's heart ... you bandage his eyes.

Overview of IGI 1 Manuscripts and Parallels: AA=BAM 20; AB=BAM 159; AC=BAM 19; AD=BAM 22; AE=BAM 18; AmA=BAM 165; AX=KAL 7/12; bA=BM 41293+; EA=EMAR 6/4; HA=STT 279; NA=BAM 510; NB=BAM 513; NC=BAM 514; ND=BAM 515; NE=BAM 516; NJ=AMT 12/5; NQ=BAM 521; NU=AMT 14/3; SA=IRAQ 65; UA=AuOrS 23/27; uA=SpTU 50; UB=Ugaritica 5; UC=AuOrS 23/14; $\mathbf{x A = C M ~} 37$ 
45’ NA i 40’ DIŠ NA IGI.MIN-šú MÚD DIRI [ ........]

NB i 31 ' ŠI]M.BI.SIG $7 .{ }^{`}$ SIG 7 ina İ.NUN? SÚD

MAR :

NC i 46’ 'DIŠ NA IGI.MIN-šú MÚD DIRI ŠIM.BI.SIG ${ }_{7}^{7}$.[SIG ${ }^{7}$ ina İ]. 'NUN SÚD $\mathrm{MAR}{ }^{\prime}$

46’ NA i 41’ úIN 6 .US Š 'tar-muš mal-ma-li[š

NB i 31' : : 'úIN.NU.UŠ` útar-mušs mal-ma-liš HE.HE i[na

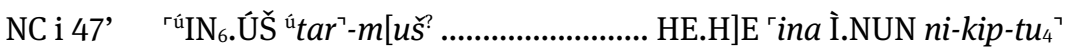
[NÍTA? $u^{?}$ MUNUS? S]ÚD 'IGI.MIN?-šú `MAR'

47’ NA i 42’ NA4 BAL ina ÚH SÚD MAR : ši $\left[m^{?}-b i^{?}-z i^{?}-d a_{4}\right.$ ?

NB ii beginning of col. ii

$\mathrm{NC}$ i 48, ${ }^{\mathrm{N}} \mathrm{NA}_{4} \mathrm{BAL}^{\urcorner}[$ .] 'ina $\mathrm{GA}^{\urcorner}$munus `Ú.ZÚG? [SÚD? $\mathrm{x}$ ] 'x HAŠHUR SÚD MAR ${ }^{7}$

48' NA i 43’ MÚD ša ŠÀ ŠAH ana ŠÀ IGI.MIN-š[ú tu-na-tak/ták ............................] NC i 49' ] x x [... IG]I.MIN-šú LAL

\begin{tabular}{|c|c|}
\hline 49’ [... ìnišu] teqqi & ${ }^{49}[. .$.$] you daub [his eyes.]$ \\
\hline
\end{tabular}

\begin{tabular}{|c|c|}
\hline 50' [... ana libbi īnišu] tazarri & 50' $[. .$.$] you spatter [. .$. into his eyes.] \\
\hline
\end{tabular}

51’ [...] sahlâ šammi ašî

52’ [...] ... ina ummāti ina mê kasî temessi 51'[...you pound] sahlû-plant, ašû-diseaseplant, ${ }^{52}[. .$.$] ... In the summer, you wash$ (the ingredients) in the sap of a kasû-

Overview of IGI 1 Manuscripts and Parallels: AA=BAM 20; AB=BAM 159; AC=BAM 19; AD=BAM 22; AE=BAM 18; AmA=BAM 165; AX=KAL 7/12; bA=BM 41293+; EA=EMAR 6/4; HA=STT 279; NA=BAM 510; NB=BAM 513; NC=BAM 514; ND=BAM 515; NE=BAM 516; NJ=AMT 12/5; NQ=BAM 521; NU=AMT 14/3; SA=IRAQ 65; UA=AuOrS 23/27; uA=SpTU 50; UB=Ugaritica 5; UC=AuOrS 23/14; xA=CM 37 


\begin{tabular}{|c|c|c|}
\hline 58 ' & $\begin{array}{l}\text { šamma peșâ }[. . .] \text { ashar ina himēti } \\
\text { tasâk teqqi }\end{array}$ & $\begin{array}{l}\text { plant, [...] x (and) ashar-stone (and) } \\
\text { daub (his eyes). }\end{array}$ \\
\hline 57' & ina GEŠTIN N[A]G? & úEME.U]R.GI ${ }_{7}$ ÍIN6.UŠ ina Zİ.KUM HE.HE \\
\hline
\end{tabular}

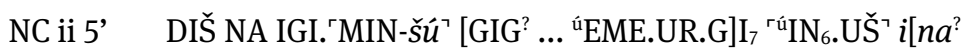
..........................]

58' NB ii 10'

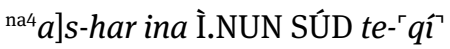

NC ii 6 ,

Ú.BABBAR ŠI[M ........................................... 'ina İ.NUN' S[ÚD ...]

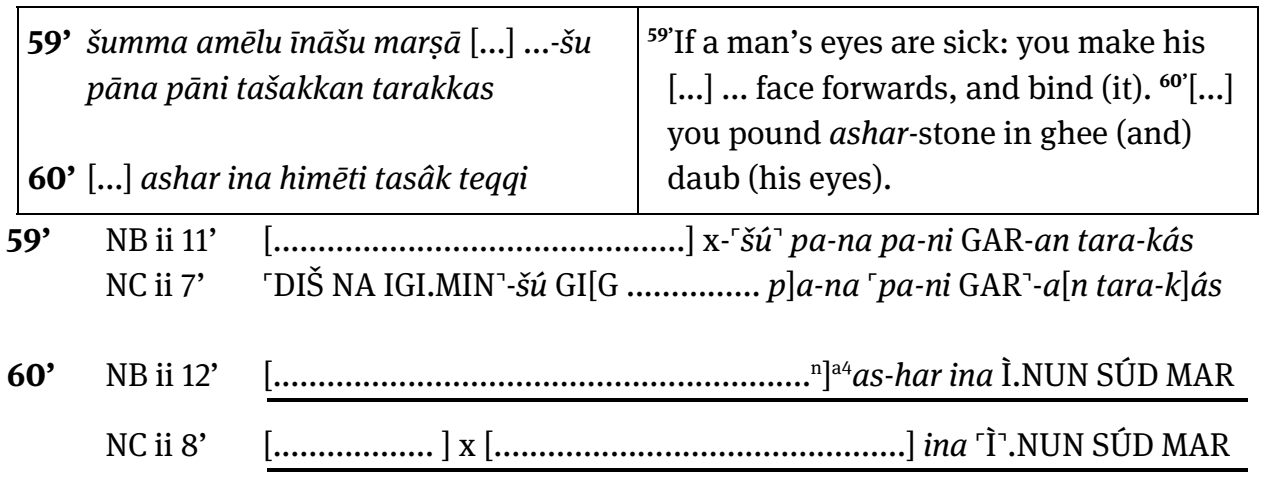

61' [šumma amēlu ìnāšu šikna] ša dāmi [šaknā (var. inațtal) šamma peșâ] rikibti arkabi emesalli nīnâ

62' [atā'iša kukra lipi kanakti] šammī annûti tamarraq ina marat immeri

63' [taballal passa teppuš šumma kūṣu ina mê šumma ummātu] ina mê kasî tasappan īnī̌u teqqi
${ }^{61}$ 'If a man's eyes have a blood deposit (var. but he is still able to see): white plant], bat guano, emesallu-saline solution, nīnû-mint, ${ }^{62}$ [atā'išu-plant, kukru-aromatic, kanaktu-aromatic pith]; you grind these drugs together (and) [ ${ }^{63}$ mix (the ingredients)] ${ }^{62}$ 'in bile of a sheep. ${ }^{63}$ 'YYou make a collyrium stick (out of it). You soften (it) in water when winter] (and) in the sap of a kasû-plant [when summer], (and) you instil his eyes (with the 'liquid').

Overview of IGI 1 Manuscripts and Parallels: AA=BAM 20; AB=BAM 159; AC=BAM 19; AD=BAM 22; AE=BAM 18; AmA=BAM 165; AX=KAL 7/12; bA=BM 41293+; EA=EMAR 6/4; HA=STT 279; NA=BAM 510; NB=BAM 513; NC=BAM 514; ND=BAM 515; NE=BAM 516; NJ=AMT 12/5; $\mathbf{N Q = B A M ~ 5 2 1 ; ~ N U = A M T ~ 1 4 / 3 ; ~ s A = I R A Q ~ 6 5 ; ~ U A = A u O r S ~ 2 3 / 2 7 ; ~} \mathbf{u A}=$ SpTU 50; UB=Ugaritica 5; UC=AuOrS 23/14; $\mathbf{x A = C M ~} 37$ 
61' NB ii 13' ..U $\left.\mathrm{U}_{5} \mathrm{~A}\right] \mathrm{RGAB} \mathrm{B}^{\text {mušen }}$

${ }^{m u n}$ eme-sal-lì úKUR.RA

NC ii 9f.' š] $\mathrm{a}$ M[ÚD $\left.\mathrm{U}_{5 .} \mathrm{ARGAB} \mathrm{B}^{\mathrm{mu}}\right]^{\mathrm{šn}}$

${ }^{m u n}$ eme-sal-lì / [úKUR.RA]

(NJ) 2f.' DIŠ NA IGI.MIN-šú ši $[k-n a$.......] x [

$$
\text { / 'rúnKUR.RA } \rightarrow
$$

(AD) r.8f.' DIŠ NA IGI.MIN-šú šik-na šá MÚD šak-'na IGI?-al 'Ú 'BABBAR

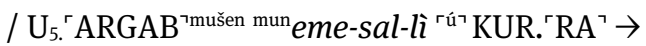

(xA) 16f.' [DIŠ NA] 'IGI.MIN-šú šik-na šá MÚD? š[ak-n]a 'Ú.BABBAR

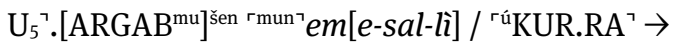

62' NB ii 14'

$$
\text { 1-niš ta-mar-raq ina ZÍ UDU.NÍTA? }
$$

NC ii 10f.' ['KU]R.K[UR .Ú HI]. $\left.{ }^{\wedge} \mathrm{A}\right\urcorner$ an-nu-ti 1-niš ta-'mar-raq / [ina ZÍ UDU.NÍTA]

(NJ) 3' rú?K[UR.KUR ] Ú.MEŠ r ̌̌EŠ $\urcorner$

(NJ) 4' UD]U.NÍTA

(AD) r.9ff.' 'úKUR`.KUR / šm GÚR.GÚR İ.UDU šm GIG 7 `Ú'.HI.A ŠEŠ 1-niš `SÚD’ / ina ZÍ UDU.NÍTA $\rightarrow$

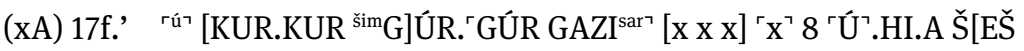
/ 'ina ZÍ UDU.NÍTA $\rightarrow$

63' NB ii 15' GAZ]I ${ }^{\text {sar }}$ ta-sa-pan IGI.MIN-šú MAR

NC ii 11' ] x ${ }^{\ulcorner}$ina A GAZI ${ }^{\mathrm{sar}\urcorner}$ ta-sa- ${ }^{\ulcorner}$pan IGI.MIN-šú MAR ${ }^{2}$

(NJ) 4' HE.HE ${ }^{r} Z^{\urcorner} \cdot \mathrm{N}[\mathrm{A}$

(AD) r.11f.' HE.HE ZA.NA DÙ-uš 'šum ${ }_{4}^{\urcorner}-m a$ É.MEŠ ina A.MEŠ / šum 4 -ma EN.TE.NA ina A.GEŠTIN.NA $t[a]$-sa-pan MAR

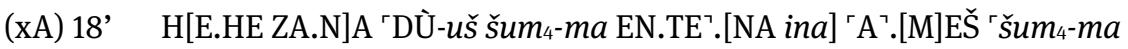
AMA.MEŠ ina A GAZI $\left.{ }^{\text {sar }} t a\right\urcorner-s[a-p a n$ MAR]

64’ šalāšat šiqil rikibti arkabi mišil šiqil šamma peșâ ina dišip šadê tasâk īnī̌u teqqi annû šammu UD.20.KAM
${ }^{64}$ 'You pound 3 shekels of bat guano, half shekel of white plant in mountain honey, daub his eyes; this is a drug (which is to be used) for twenty days.

Overview of IGI 1 Manuscripts and Parallels: AA=BAM 20; AB=BAM 159; AC=BAM 19; AD=BAM 22; AE=BAM 18; AmA=BAM 165; AX=KAL 7/12; bA=BM 41293+; EA=EMAR 6/4; HA=STT 279; NA=BAM 510; NB=BAM 513; NC=BAM 514; ND=BAM 515; NE=BAM 516; NJ=AMT 12/5; NQ=BAM 521; NU=AMT 14/3; sA=IRAQ 65; UA=AuOrS 23/27; uA=SpTU 50; UB=Ugaritica 5; UC=AuOrS 23/14; xA=CM 37 
64' NA ii 1' 3 GÍN ' $\mathrm{U}_{5}$ ' .[ARGAB ${ }^{\text {mušen }}$

NB ii 16 , SÚD IGI.MIN-šú MAR an-nu-u šam-mi UD.20.KÂM

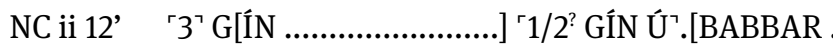
SÚ]D IGI.MIN-šú MAR 'an'-nu Ú UD.20.KÁM

65' šiptu ib-da-gub numun [... ina] kīri balāti

66’ balātī napšir balātī napšir

67’ ba'lat ina itti amallik [...] sinništu ul ullad akla mēșa mādu tê šipti

68’ ka'inimma īnāšu dāma malâ

${ }^{65}$ 'Incantation: the seed stays with it [........ in the] 'kiln of life', ${ }^{66}$ 'My life be saved; my life be saved!' ${ }^{67}$ 'She is too large; I can judge from the symptom [... (this)] woman cannot give birth. I hold back (treatment) completely. Incantation spell. ${ }^{68}$ 'Invocation (for the case if) his eyes are full of blood.

65' NA ii 2' ÉN ib-da-g[ub

NB ii 17 , $k] i^{2}-{ }^{r} i-r i b a-l a-t \underline{i}$

NC ii 13’ ÉN ib-'da-gub numun? ${ }^{?}[$. in] $\left.a^{?}{ }^{\ulcorner} k i-i\right\urcorner-r i b a-\ulcorner l a-t ̦ i\urcorner$

66' NA ii 3' ba-la-ți n[a-ap-ši-ir ba-l]a-'țì na'-[ap-ši-ir]

NB ii 18, [............................. ba-la]-ṭi ${ }^{\top} n a^{\top}-a p$-ši-ir

NC ii 14' ba-la-ți na-ap-ši-i $[r$...............na- na $] p$ - ‘̌̌i-ir

67' NA ii 4' ba-'-la-at ina it-ti [ MU]NUS? $\left.{ }^{r} \mathrm{NU}\right\urcorner \mathrm{U} . \mathrm{TU}$

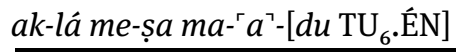

NB ii 19' x $\left.{ }^{r} \mathrm{NU}\right\urcorner \mathrm{U} . \mathrm{TU}$ $\ulcorner a k+l a ́ m e\urcorner-s ̣ a m a-a-d u \mathrm{TU}_{6}$.ÉN

NC ii 15, ba-'-la-at ina it-ti a-ma-li[ $k^{\text {? }}$ $\left.\mathrm{TU}_{6} . \mathrm{E}\right] \mathrm{N}$

68' NA ii 5’ KA.INIM.[MA IG]I.MIN-šú MÚD DI[RI.MEŠ]

NB ii 20' ] MÚD DIRI.MEŠ

Overview of IGI 1 Manuscripts and Parallels: AA=BAM 20; AB=BAM 159; AC=BAM 19; AD=BAM 22; AE=BAM 18; AmA=BAM 165; AX=KAL 7/12; bA=BM 41293+; EA=EMAR 6/4; HA=STT 279; NA=BAM 510; NB=BAM 513; NC=BAM 514; ND=BAM 515; NE=BAM 516; NJ=AMT 12/5; NQ=BAM 521; NU=AMT 14/3; SA=IRAQ 65; UA=AuOrS 23/27; uA=SpTU 50; UB=Ugaritica 5; UC=AuOrS 23/14; xA=CM 37 


\begin{tabular}{|c|c|c|c|}
\hline \multicolumn{3}{|c|}{$\begin{array}{l}\text { 69’ dudubi šuruš urâni ... uqnâta tarakkas } \\
\text { erbēšeret kișrī takașṣar ēma takașṣaru } \\
\text { šipta tamannu ina nakkaptī̌su } \\
\text { tarakkas }\end{array}$} & $\begin{array}{l}{ }^{69} \text { 'Its medical application: you bind } \\
\text { (together) root of urânu-plant, [...], and } \\
\text { uqnâtu-blue-plant, (and) make fourteen } \\
\text { knots. Wherever you make a knot you } \\
\text { shall recite the incantation (see ll. 65ff.' } \\
\text { above), (and) you bind it on his temple. }\end{array}$ \\
\hline \multirow[t]{3}{*}{ 69' } & NA ii 6' & \multicolumn{2}{|c|}{$\begin{array}{l}\text { DÙ.DÙ.BI SUHU[Š ........................................................... } 14 \\
\text { K]A.KEŠDA KEŠDA e-ma KEŠDA ÉN ŠID-nu ina SAG.K[I-šú ...............] }\end{array}$} \\
\hline & NB ii 21' & \multicolumn{2}{|c|}{ KlA rKFŠDA } \\
\hline & NC ii 17’ & \multicolumn{2}{|c|}{$\begin{array}{l}\text { DÙ.DÙ.BI SUHUŠ úr HA SAHAR? } \mathrm{x} \text { ú? ZA.GÌN.NA tara-kás } 14 \\
\text { KA.KE[ŠDA }\end{array}$} \\
\hline \multicolumn{3}{|c|}{$\begin{array}{l}\text { 70’ šiptu huhahi lā bāmâ huhahi lā bāmâa } \\
\text { u agašgû uqatta agašgû uqatta tê šipti }\end{array}$} & $\begin{array}{l}{ }^{70} \text { Incantation: (The syllables) hu-ha-hi: } \\
\text { not by halves! hu-ha-hi: not by halves! } \\
\text { Even a novice can complete it (the } \\
\text { syllabary). A novice can complete it! } \\
\text { Incantation spell. }{ }^{71} \text { Invocation: if a } \\
\text { man's eyes are full of blood. }\end{array}$ \\
\hline \multirow[t]{3}{*}{$70^{\prime}$} & NA ii 7 & \multicolumn{2}{|c|}{ 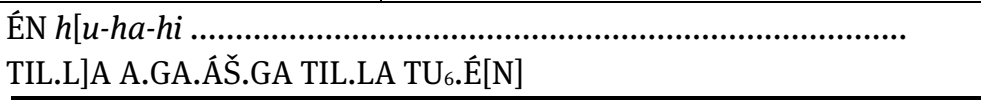 } \\
\hline & NB ii 22’ & \multicolumn{2}{|c|}{ 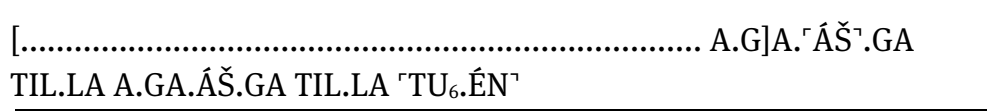 } \\
\hline & NC ii 18’ & \multicolumn{2}{|c|}{ 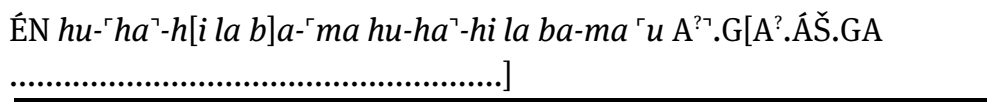 } \\
\hline \multirow[t]{3}{*}{$71^{\prime}$} & NA ii 8 ' & \multicolumn{2}{|c|}{ 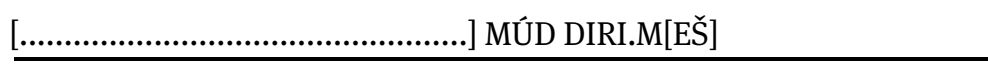 } \\
\hline & NB ii $23^{\prime}$ & \multicolumn{2}{|c|}{ [................................] IGI.MIN-šú 'MÚD DIRI.MEŠ` } \\
\hline & NC ii 19' & \multicolumn{2}{|c|}{ 'KA.INIM.MA DIŠ NA IGI.MIN’-šú [.........................] } \\
\hline
\end{tabular}

Overview of IGI 1 Manuscripts and Parallels: AA=BAM 20; AB=BAM 159; AC=BAM 19; AD=BAM 22; AE=BAM 18; AmA=BAM 165; AX=KAL 7/12; bA=BM 41293+; EA=EMAR 6/4; HA=STT 279; NA=BAM 510; NB=BAM 513; NC=BAM 514; ND=BAM 515; NE=BAM 516; NJ=AMT 12/5; NQ=BAM 521; NU=AMT 14/3; sA=IRAQ 65; UA=AuOrS 23/27; uA=SpTU 50; UB=Ugaritica 5; UC=AuOrS 23/14; xA=CM 37 


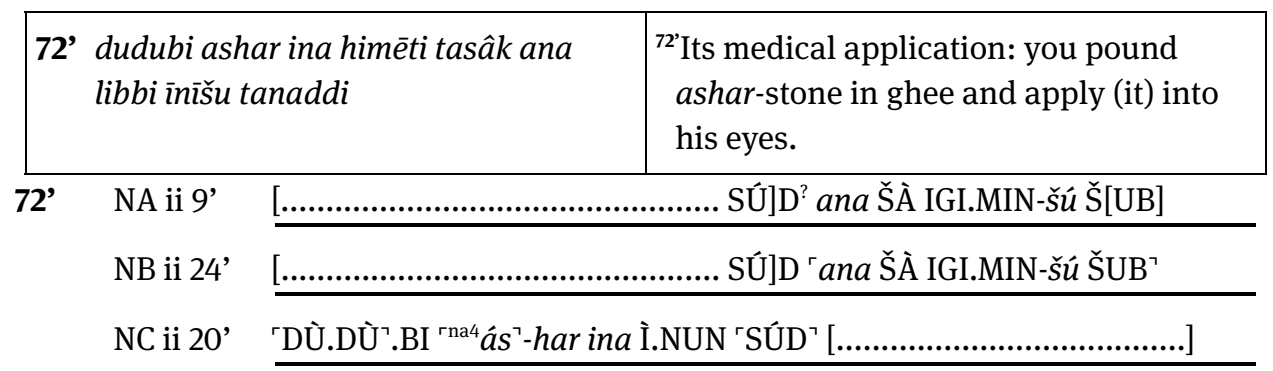

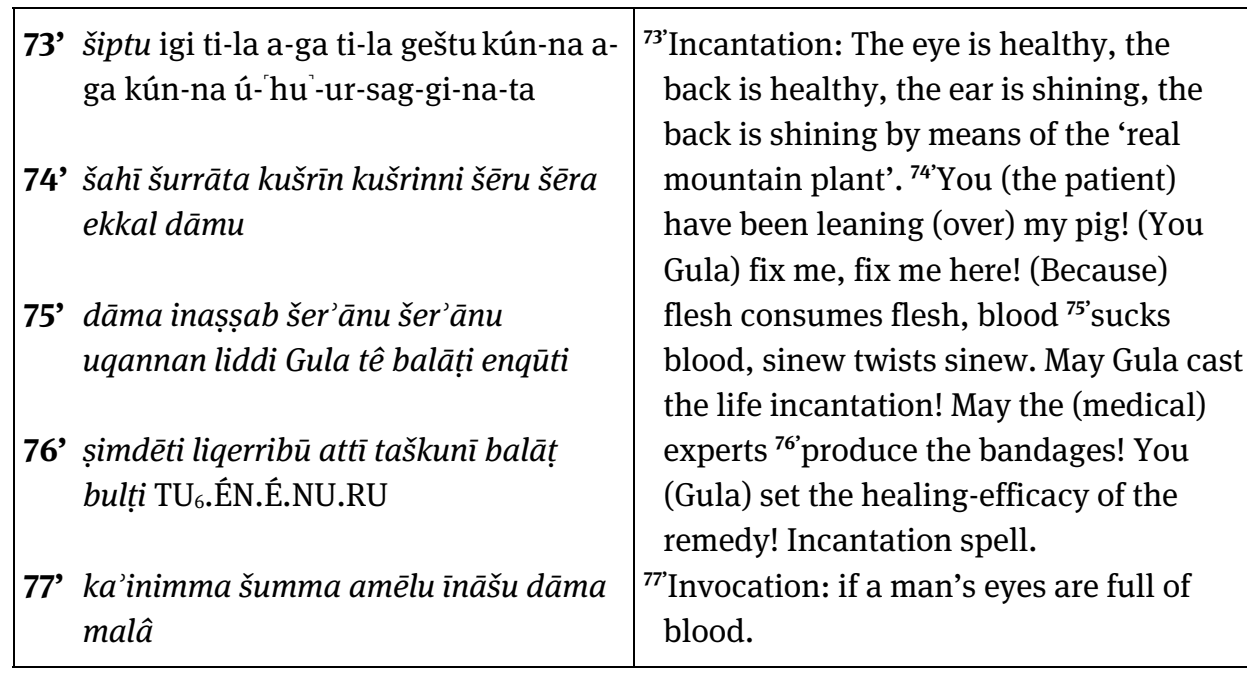

73' NA ii 10' [................................................... kún-n]a ú-'hu-ur-sag ${ }^{\urcorner}-g[i-n a-t a]$

NB ii 25’ [...................................................... kún-n]a'ú-hu-ur-sag-gi '-na-ta

NC ii 21' ÉN igi ti-la a-ga ti-la geštu kún?-na a-ga kún-na ú-`hu'-[ur-sag-gi-nata]

(UA) r.7f. [ÉN igi t]i-la a-ga ti-[la] / [...] $\quad x \quad x \quad[\ldots] /[\ldots]$ gi-na-ta $[\ldots]$

74' NA ii 11'

NB ii 26,

še-e-ru še-e-ra e-k[al ...........]

NC ii 22' ša-hi šur-ra-ta kuš-ri-in kuš-ri-in-ni še-e-ru še-e-r $[a$

75' NA ii 12'

Š]UB- $d i{ }^{\mathrm{d}} g u-l a \mathrm{TU}_{6}$

'TI '[LA .............]

Overview of IGI 1 Manuscripts and Parallels: AA=BAM 20; AB=BAM 159; AC=BAM 19; AD=BAM 22; AE=BAM 18; AmA=BAM 165; AX=KAL 7/12; bA=BM 41293+; EA=EMAR 6/4; HA=STT 279; NA=BAM 510; NB=BAM 513; NC=BAM 514; ND=BAM 515; NE=BAM 516; NJ=AMT 12/5; NQ=BAM 521; NU=AMT 14/3; SA=IRAQ 65; UA=AuOrS 23/27; uA=SpTU 50; UB=Ugaritica 5; UC=AuOrS 23/14; xA=CM 37 
NB ii 27’ ú-qa-an-n]a-an ŠUB-di ${ }^{\mathrm{d}} g u-l a \mathrm{TU}_{6}$

TI.LA 'en?-qu-ti

NC ii 23’ da-ma i-na-șab SA-a-nu SA-a-nu ú-qa-an-na-an ŠUB-di ${ }^{\mathrm{d}}$ gu-la TU6 'TI'.L[A ..]

76' NA ii 13' b]a-laṭ bu-ul-ți $\mathrm{TU}_{6} . \mathrm{E} \mathrm{N}[$.

NB ii 28' taš-ku]n? ba-laṭ bu-ul-ṭ̂́ TU.Én.É.NU.RU

NC ii 24' și-im-de-ti li-qer-'ri’-bu at-ti taš-ku-ni ba-laț bu-ul-ți TU6.ÉN.É.N[U.RU]

77’ NA ii 14' N]A IGI.MIN-šú MÚD DI[RI.MEŠ]

NB ii 29' ..] IGI.MIN-šú MÚD DIRI.MEŠ

NC ii 25 , KA.INIM.MA DIŠ NA IGI.MIN-šú MÚD DIRI.[MEŠ]

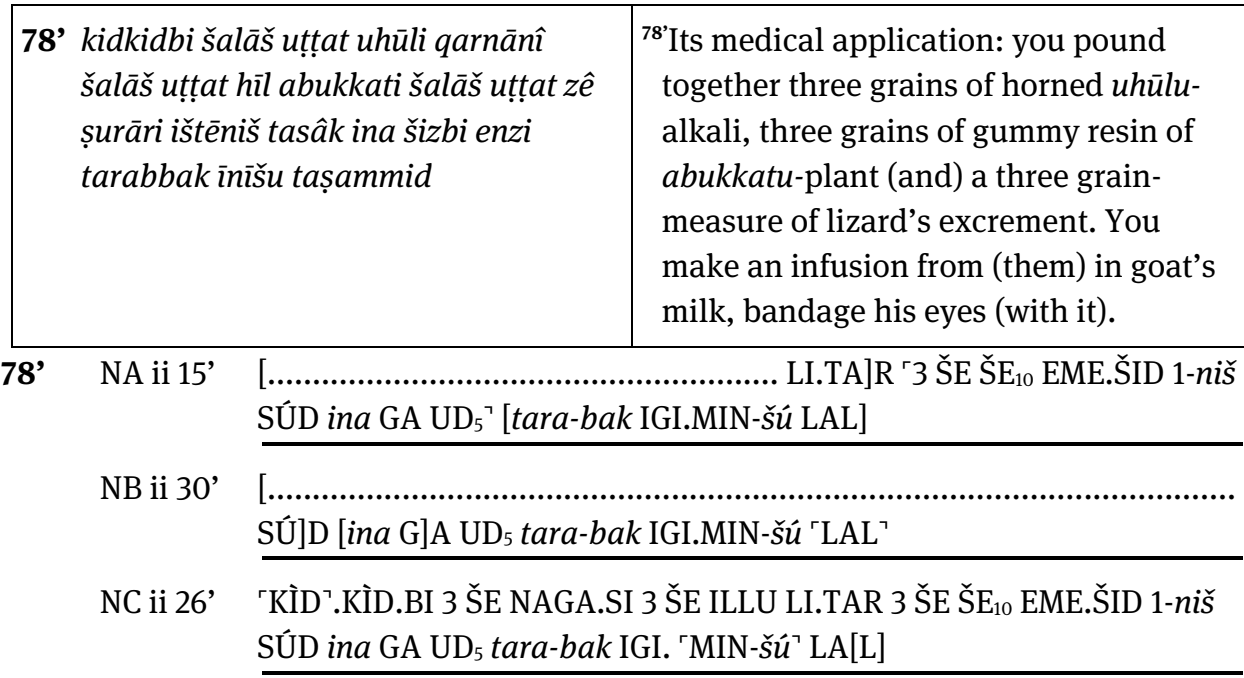

79’ šumma amēlu īnāšu marșāma dāma malâ baluhhī dāma ultatanni'ā dāmu dimtu ina libbi īnišu ittașâ
${ }^{79}$ 'If a man's eyes are sick and then full of blood. They (= the eyes) are suffused with baluhhu-granules (and) blood, blood (and) tear(s) come out from the middle of his eyes. ${ }^{80} \mathrm{~A}$ film pushes away

Overview of IGI 1 Manuscripts and Parallels: AA=BAM 20; AB=BAM 159; AC=BAM 19; AD=BAM 22; AE=BAM 18; AmA=BAM 165; AX=KAL 7/12; bA=BM 41293+; EA=EMAR 6/4; HA=STT 279; NA=BAM 510; NB=BAM 513; NC=BAM 514; ND=BAM 515; NE=BAM 516; NJ=AMT 12/5; NQ=BAM 521; NU=AMT 14/3; SA=IRAQ 65; UA=AuOrS 23/27; uA=SpTU 50; UB=Ugaritica 5; UC=AuOrS 23/14; $\mathbf{x A = C M ~} 37$ 
80’ șillu lamassat īnīšu unakkap ašìtu ana șilli itūr digal ikabbitūšu bīna arqūssu tuhassa

81' ina țābāti dannāti tarammuk ina kakkabi tušbât ina šèri ana libbi huliam taṣahhat

82’ alluhara šamma peșâ emesalli lipâ tuškâ zība hīl erî ahennû tasâk

83’ malmališ teleqqe ištēniš tuštemmid ana libbi huliam ša tašhutu tašappak

84' ina himēti u šumēni talâšma rēš īnišu ina ubāni tepette ana libbi īnišu tašakkan

85’ ināšu katmā ukâl iniñšu takârma UD.9.KAM annâ tēteneppuš

\section{Alternative prescription 1}

86' ana ašri šanîmma murra šamma peșâ emesalli ina uppi siparri ana libbi inišu tanappah

\section{Alternative prescription 2}

87’ šumma amēlu šanîš hīl erî šamma peșâ tasâk ina uppi siparri ana libbi inišu tanappah the pupil of his eyes. The blurred vision has turned into a film, with seeing being burdensome to him. You squeeze binutamarisk while still fresh (and) ${ }^{81}$ 'soak (it) in strong vinegar and let it stay under a star (= stay overnight). In the morning, you press it out into a funnel (lit. huliam-vessel). ${ }^{82}$ You pound separately alluharu-mineral, white plant, emesallu-saline solution, fat, tuškû-mineral, black cumin (and) exudation of copper. ${ }^{83}$ You take in equal amounts, stir (them) together. You pour (them) into the funnel (lit. huliamvessel), which you have (earlier) rinsed. ${ }^{84}$ 'You knead (it) in ghee and šumēnumineral, then you open the upper part of his eyes with a finger (and) put (the medication) into his eyes. ${ }^{85} \mathrm{He}$ keeps his eyes closed (while) you rub his eyes (i.e. the eyelids and surroundings) and continuously do this for nine days.

\section{Alternative prescription 1}

${ }^{86}$ Alternatively: you blow bitter-plant, white plant (and) emesallu-saline solution with a bronze tube into his eyes.

\section{Alternative prescription 2}

${ }^{87}$ 'If a man: alternatively, you pound exudation of copper (and) white plant, you blow it with a bronze tube into his eyes.

Overview of IGI 1 Manuscripts and Parallels: AA=BAM 20; AB=BAM 159; AC=BAM 19; AD=BAM 22; AE=BAM 18; AmA=BAM 165; AX=KAL 7/12; bA=BM 41293+; EA=EMAR 6/4; HA=STT 279; NA=BAM 510; NB=BAM 513; NC=BAM 514; ND=BAM 515; NE=BAM 516; NJ=AMT 12/5; NQ=BAM 521; NU=AMT 14/3; sA=IRAQ 65; UA=AuOrS 23/27; uA=SpTU 50; UB=Ugaritica 5; UC=AuOrS 23/14; $\mathbf{x A = C M ~} 37$ 


\section{Alternative prescription 3}

88' šumma amēlu šanîš urnê šamma peșâ tasâk ina uppi siparri ana libbi īnīšu tanappah

DIŠ NA IGI. 'MIN ${ }^{\urcorner}-\check{s}[u$ $u l-t a-t] a-$ ni-'a MÚD ÉR ina ŠÀ IGI.MIN-šú 'È’-[a]

NB ii 31'

Š] À IGI.MIN-šú 'È- $a^{\urcorner}$

NC ii 27’ [DIŠ N]A IGI.MIN-šú GIG-ma MÚD DIRI šmBULUH.HI.A MÚD ul-ta-tani-'a MÚD ÉR ina ŠÀ IGI.MIN-šú 'È’-[a]

80’ NA ii 17’ GISSU dr LAMA ${ }^{\urcorner}$IG[I.MIN-šú GISSU GU]R di-gal 9 DUGUD-šú giššINIG SIG ${ }_{7}-s u$ tu-[ha-sa]

NB ii 32'

$$
\text { gišŠI]NIG }{ }^{r} \mathrm{SIG}_{7}-\mathrm{Su} \text { tu-ha-sa }{ }^{\urcorner}
$$

NC ii 28’ [GIS]SU dLAMA IGI.MIN-šú ú-na-kap a-ši-tu ana GISSU GUR 'di`-gal, 'DUGUD?-šú giššINIG SIG ${ }^{7}-s u$ tu-ha-s[a]

81’ NA ii 18' ina A.GEŠTIN.NA KALA.GA $\operatorname{tar}[a-m u k$ Á.G]Ú.ZI.GA

NB ii 33' ana ŠÀ hu-li-ia-am 'SUR?'-[at]

NC ii 29' [ina A.GE]ŠTIN.NA KALA.GA tara-muk ina UL tuš-bat ina Á.GÚ.ZI.GA ana ŠÀ hu-li-ia-am SUR- 'at ${ }^{\urcorner}$

(NU) 7’ S]IG-su SÚD A.MEŠ-šú SUR-at ina UL tuš-bat KIMI[N]

82' NA ii 19' IM.SAHAR.BABBAR.KUR.RA Ú.BABBAR ${ }^{\mathrm{mu}[\mathrm{n}}$ İ.UD]U

NB ii 34'

$$
\text { tuš-ka- } a \text { úzi-ba- } a \text { ILLU 'URUDU } a-h e^{\urcorner}-[n u-u ́ ~ . . .]
$$

NC ii 30’ [IM.SAHAR].BABBAR.KUR.RA Ú.BABBAR ${ }^{\text {rmun }}$ eme-sal-lì İ.UDU tuš-ka- $a\urcorner$ úzi-ba- $a$ ILLU URUDU $a-h e-n u-u$ SÚD

83’ NA ii 20' mál-ma-liš TI-qé 1-niš tuš-te-mid an[a ŠÀ hu-li-ia-a]m ša ta-áš-hu-tu DUB-[ak]

Overview of IGI 1 Manuscripts and Parallels: AA=BAM 20; AB=BAM 159; AC=BAM 19; AD=BAM 22; AE=BAM 18; AmA=BAM 165; AX=KAL 7/12; bA=BM 41293+; EA=EMAR 6/4; HA=STT 279; NA=BAM 510; NB=BAM 513; NC=BAM 514; ND=BAM 515; NE=BAM 516; NJ=AMT 12/5; NQ=BAM 521; NU=AMT 14/3; sA=IRAQ 65; UA=AuOrS 23/27; uA=SpTU 50; UB=Ugaritica 5; UC=AuOrS 23/14; xA=CM 37 
NB ii 35, $h u-l] i-\left\ulcorner i a^{\urcorner}-a m\right.$ ša ta-áš-hu-tu DUB- $a k$

NC ii 31' [.............] TI-qé 1-niš tuš-te-mid ana ŠÀ hu-li-ia-am 'ša ta-áš-hu-tu DUB- $a k^{?}$

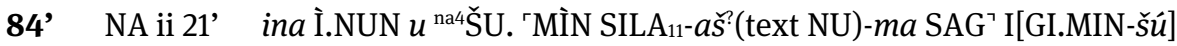
ina ŠU.SI BAD-te ana ŠÀ IGI.MIN-šú GAR-[an]

NB ii 36' IGI.MIN-š]ú ina ŠU.SI BAD-te ana ŠÀ IGI.MIN-šú GAR-an

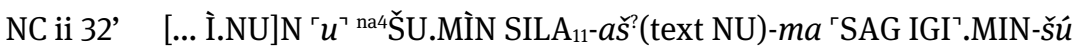
ina ŠU.'SI BAD-te ana ŠÀ IGI.MIN-šú GAR-an

85' NA ii 22’ IGI.MIN-šú DUL-ma ú-kal IGI.MIN-šú ta-kar-'ma UD?.9.KÁM an-na-a DÙ.[MEŠ]

NB ii 37'

$[\ldots$ IGI.MI]N-šú ta-kar-ma UD.9.KAM* an-na-a DÜ.MEŠ

NC ii 33’ DU]L-ma ú-kal IGI.MIN-šú 'ta-kar-ma? UD.9.KÁM an-na-a DÙ.MEŠ

86’ NA ii 23’ DIŠ KIMIN ${ }^{\text {ršim? }}{ }^{\prime}$ SES Ú.BABBAR ${ }^{\text {mun }}$ eme-sal-lì [ina MU]D ZABAR ana ŠÀ IGI.MIN-šú B[ÚN?]

NB ii 38' $\left.{ }^{\mathrm{mu}}\right]^{\mathrm{n}}$ eme-sal-lì ina MUD Z[ABAR ana ŠÀ IGI].MI[N-šú BÚN]

NC ii 34' SE]S Ú.BABBAR ${ }^{\text {mun }}$ eme-sal-lì ina MUD ZABAR ana ŠÀ IGI.MIN-šú BÚN

87’ NA ii 24’ DIŠ NA MIN ILLU URUDU Ú.BABBAR SÚD [ina MU]D 'ZABAR ana ŠÀ IGI.MIN-šú [BÚN]

NB ii 39'

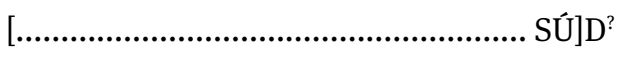

..]

NC ii 35' ILL]U? `URUDU Ú’.BABBAR SÚD ina MUD ZABAR ana ŠÀ IGI.MIN-šú BÚN

Overview of IGI 1 Manuscripts and Parallels: AA=BAM 20; AB=BAM 159; AC=BAM 19; AD=BAM 22; AE=BAM 18; AmA=BAM 165; AX=KAL 7/12; bA=BM 41293+; EA=EMAR 6/4; HA=STT 279; NA=BAM 510; NB=BAM 513; NC=BAM 514; ND=BAM 515; NE=BAM 516; NJ=AMT 12/5; NQ=BAM 521; NU=AMT 14/3; sA=IRAQ 65; UA=AuOrS 23/27; uA=SpTU 50; UB=Ugaritica 5; UC=AuOrS 23/14; $\mathbf{x A = C M ~} 37$ 
88’ NA ii 25’ 'DIŠ` NA MIN úúr-né-e Ú.BABBAR SÚD `ina MUD ZABAR ${ }^{\prime}$ ana ŠÀ IGI.MIN-šú [BÚN]

NB ii 40,

$[$.. S]ÚD `ina MUD? ZABA[R

NC ii 36'

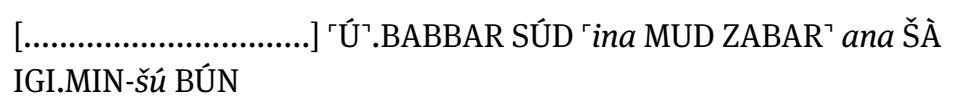

89’ šiptu igi bar igi bar-bar igi bar-ra barbar igi huš igi huš-huš igi bar-ra hušhuš

90’ igi bar ná-a igi bar da-a igi bar hul-a ìnā abâtu ìnā ašâtu

91' [pursìt] dāmi šuharratu ìnā kīma niksi immeri dāma šenâ

92’ kīma mê ša agalapê alapâ nadâ kīma karpat țābāti nadâ șilla

93’ ina berišina pitiqtu patqat īrub Šakkan ina berūšina ittadi kussâšu

94’ aššum annitu ina libbi annìte lā napāše šiptu ul yā'uttun šipat Ea u Asalluhi

95’ šipat Dāmu u Gula šipat Ningirimma bēlet šiptī Gula bullițīma qištaki liqī tê šipti

96’ ka'inimma īni marušti
${ }^{89}$ Incantation: the open eye is a staring eye, the opened eye stares, the reddish eye is a crimson eye, the opened eye is crimson. ${ }^{90}$ The open eye is drowsy, the open eye is weak, the open eye is harmed. O cloudy eyes, blurred eyes (vision). ${ }^{91}$ [The (eye)] blood [vessel] is porous. The eyes are suffused with blood like a slaughtered sheep, ${ }^{92}$ they (= the eyes) are spotted like the (muddy) water of a lagoon with alapû-algae, they (= the eyes) are spotted like a vinegar-jar with a film. ${ }^{93}$ In between them (= the eyes), a mud-brick wall is constructed (= nose). (The god) Šakkan entered between them (= the eyes) and installed his seat. ${ }^{94}$ Therefore, one can not breathe in the middle of this (nose). The incantation is not mine; it is the incantation of Ea and Asalluhi, ${ }^{95}$ 'the incantation of Dāmu and Gula, the incantation of Ningirimma, mistress of incantations. O Gula keep (me) healthy and accept your fee. Incantation spell. ${ }^{96}$ It is an invocation for a sick eye.

89’ NA ii 26’ ÉN igi bar igi bar-bar igi bar-ra bar-bar 'igi huš ${ }^{\prime}$ igi huš-huš igi bar-ra $\mathrm{h}[$ uš-huš]

Overview of IGI 1 Manuscripts and Parallels: AA=BAM 20; AB=BAM 159; AC=BAM 19; AD=BAM 22; AE=BAM 18; AmA=BAM 165; AX=KAL 7/12; bA=BM 41293+; EA=EMAR 6/4; HA=STT 279; NA=BAM 510; NB=BAM 513; NC=BAM 514; ND=BAM 515; NE=BAM 516; NJ=AMT 12/5; NQ=BAM 521; NU=AMT 14/3; sA=IRAQ 65; UA=AuOrS 23/27; uA=SpTU 50; UB=Ugaritica 5; UC=AuOrS 23/14; xA=CM 37 
NB ii 41' b]ar-ra bar-bar igi huš igi huš-huš igi bar-ra $\mathrm{h}[$ uš-huš]

NC ii 37' bar-r]a bar-bar igi huš igi huš-huš igi bar-ra 'huš-huš

(UA) 5’ [én igi h]uš igi huš-huš igi bar-ra hu[š-huš ....]

90’ NA ii 27’ [igi b]ar `ná-a igi bar da-a igi bar hul'-a IGI.MIN $a$-ba-tu IGI.MIN $a-[\check{s} a-t u]$

NB ii 42' d]a-a igi bar hul-a IGI.MIN $a$-ba-tu IGI.MIN $a-\ulcorner\check{s} a\urcorner-[t u]$

NC ii 38' da]-`a’ igi bar hul-a IGI.MIN $a$-ba-tu IGI.MIN $a-\left\ulcorner\check{s} a^{\urcorner}-[t u]\right.$

91' NA ii 28' šu-h]ar-ra-tú IGI.MIN ${ }^{\text {ERASURE }} \mathrm{GIN}_{7}$ nik-si UDU.NÍTA MÚD NB ii 43'<smiles>I[Tl]</smiles>

$[\ldots$

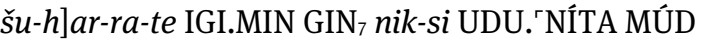
še-en- $a^{\urcorner}$

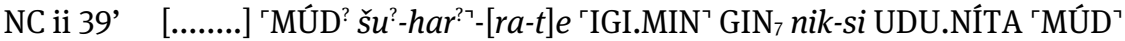
š $[e-e n-a]$

(NC) iii 39' 'ÉN' [ IGI.MIN pur-s]i-`it? MÚD šu-har-ra-tu

(UB) 4f. IGI.MEŠ pur-sí-it 'MÚD'.ME[Š] / šu-har-re-tu

(EA) 3 pur-si-it da-mi i-na-šs $[u]$

92' NA ii 29' .] ' $a\urcorner-l a-p a-a$ ŠUB- $a$ ki-ma DUG A.GEŠTIN.NA ŠUB- $a[\ldots . . . .$.

NB ii 44' $a$-la-p]a- $a$ ŠUB- $a$ ki-ma DUG A.GEŠTIN.NA 'ŠUB- $a$ ' șil-la

NC ii 40’ 'rGIN 7 A.MEŠ šá a-gala-pe-e a-l[a-pa-a .... ki-m]a DUG 'A.GEŠTIN.NA` [.....................]

93' NA ii 30’ pat-qa]t? $\left.{ }^{\mathrm{K}} \mathrm{KU}_{4}-u b\right\urcorner \mathrm{d} \mathrm{GÌR}$ ina be-ru-ši-na it-ta-[di NB ii 45' pi-ti-i]q-tu ${ }_{4}$ pat-qat $\mathrm{KU}_{4}-u b{ }^{\mathrm{d}} \mathrm{GÌR}$ ina be-ru-ši-na it-ta-di gišGU.ZA-šú

NC ii 41' ina be-ri-ši-na pi-ti-iq-tu ${ }_{4}$ pat-q[at be-ru-š $] i-n[a$

Overview of IGI 1 Manuscripts and Parallels: AA=BAM 20; AB=BAM 159; AC=BAM 19; AD=BAM 22; AE=BAM 18; AmA=BAM 165; AX=KAL 7/12; bA=BM 41293+; EA=EMAR 6/4; HA=STT 279; NA=BAM 510; NB=BAM 513; NC=BAM 514; ND=BAM 515; NE=BAM 516; NJ=AMT 12/5; $\mathbf{N Q = B A M ~ 5 2 1 ; ~ N U = A M T ~ 1 4 / 3 ; ~ s A = I R A Q ~ 6 5 ; ~ U A = A u O r S ~ 2 3 / 2 7 ; ~} \mathbf{u A}=$ SpTU 50; UB=Ugaritica 5; UC=AuOrS 23/14; $\mathbf{x A = C M} 37$ 
(UC) r.14 x i-na be-ri-šu pi-ti ${ }_{4}-i k-t u_{4} p a-a t-k a-a t \mathrm{TU}_{6}$.ÉN.É.NU. ${ }^{r} \mathrm{RU}{ }^{\prime}$

94’ NA ii 31' É]N ‘ul' ia-at-tú-un ÉN ${ }^{\mathrm{d}} e-a\ulcorner u\urcorner[\ldots \ldots \ldots . . . .$.

NB ii 46, ] la na-pa-še ÉN ul `ia-at-tu-un? ÉN ${ }^{\mathrm{d}}$ e- $a$ u ${ }^{\mathrm{d}}$ asal-lú-hi

NC ii 42’ áš- '̌šum an-ni-tu ina ŠÀ an-ni-te la na-pa-še É[N?

(EA) 13 ši-ip-tu ú-ul i-ia-tù

95' NA ii 32' NÍG.BA- $k[i$............. $]$

NB ii 47'

$[\ldots$ ${ }^{\mathrm{d}}$ gu-l]a ÉN ${ }^{\mathrm{d}}$ nin-gìrima be-let šip-te ${ }^{\mathrm{d}}$ gu-la TI-ma NÍG.BA-ki TI TU 6 .ÉN

NC ii 43' ÉN ${ }^{\mathrm{d}} d a-m u u^{\mathrm{d}}$ gu-la 'ÉN ${ }^{\mathrm{d}}$ nin-gìrima ${ }^{\top}$ [

(EA) 13f. $\quad$ ši-pa-at ${ }^{\mathrm{d}} d a-m u / u^{\mathrm{d}} g u-l a$

96' NA ii 33' ] IGI GIG.GA.[.........]

NB ii 48, ] IGI GIG.GA.A.KÁM

NC ii 44’

[......................] IGI GIG.GA.A.KÁM

97’ dudubi annû ša šīpāti peșâti țurra tațammi sebet kiṣrī takașșar ēma takașșaru šipta tamannu ina īnī̌u balitti tarakkassu
${ }^{97}$ Its medical application: you spin this which is a cord of white wool. You shall tie (on) seven knots. Whenever you make a knot, you shall recite the (above) incantation, and bind it on his healthy eye!

\section{7' NA ii 34'}

Overview of IGI 1 Manuscripts and Parallels: AA=BAM 20; AB=BAM 159; AC=BAM 19; AD=BAM 22; AE=BAM 18; AmA=BAM 165; AX=KAL 7/12; bA=BM 41293+; EA=EMAR 6/4; HA=STT 279; NA=BAM 510; NB=BAM 513; NC=BAM 514; ND=BAM 515; NE=BAM 516; NJ=AMT 12/5; NQ=BAM 521; NU=AMT 14/3; sA=IRAQ 65; UA=AuOrS 23/27; uA=SpTU 50; UB=Ugaritica 5; UC=AuOrS 23/14; xA=CM 37 
NB iii 1 $a n-n u]-\ulcorner u ́$ ša SÍG BABBAR DUR NU.NU 7 KA.KEŠDA KEŠDA ${ }^{\urcorner} e-m a$ KEŠDA ÉN ŠID-nu ina IGI-šú ${ }^{\top T I}$.LA-ti KEŠDA-su?

NC ii 45f.' 'DÙ.DÙ?.BI an-nu-u ša SÍG BABBAR DUR NU.NU `7? [ .] / ina IGI-š[ú

98’ šiptu igi bar igi bar-bar igi bar-ra barbar igi huš igi huš-huš igi bar-ra hušhuš

99’ [igi bar ná-a igi] bar da-a igi bar hul-a ìnā ap/bâtu ìnā ašâtu ìnā ša dāma malâ

100’ [anāku ...] ... sāniq qabû ša Gula

101’ šittama šinama ahhātu ina berūšina parik šadû

102’ manna lušpur ana mārat Ani ša šamê

103’ liššâni tallišina hulāli karpātīšina uqnî ebbi lisāpāni mê ayabbi

104' tâmti rapašte ša harištu lā ūridu ana libbi musukkati lā imsû qātīša

105’ lislânimma/limlânimma likeșṣ̂a umma șirihta ša libbi ìnī̌u

106' šiptu ul yā'uttun šipat Ea u Asalluhi šipat Dāmu u Gula

107’ šipat Ningirimma bēlet šipte Gula bullițīma qištaki liqì tê šipti 108' ka'inimma īni marušti
${ }^{98}$ Incantation: The open eye is a staring eye, the opened eye stares, the reddish eye is a crimson eye, the opened eye is crimson. ${ }^{99}$ [The open eye is drowsy], the open [eye] is weak, the open eye is harmed (lit. evil). $\mathrm{O}$, cloudy eyes, $\mathrm{O}$ blurred eyes, eyes which are full of blood. ${ }^{100}[\mathrm{I}$ am .... ... the one who executes the command of Gula. ${ }^{101}$ 'They are two, the two are sisters (= the eyes), between whom a mountain is parked! ${ }^{102}$ 'Whom shall I send to the celestial daughter of Anu? ${ }^{103}$ 'May they bring forward their flasks (made) from banded agate (onyx), their vessels (made) from pure lapis lazuli, and draw water of the sea ${ }^{104}$ 'and ocean, into which no woman in confinement plunged (and) no woman in maternity washed her hands! ${ }^{105}$ 'May they sprinkle (var. fill water) and cool off the ummu-fever (and) the hotness midst of his (patient) eyes. ${ }^{106}$ 'The incantation is not mine. It is the incantation of Ea and Asalluhi, the incantation of Dāmu and Gula, ${ }^{107}$ the incantation of Ningirimma, mistress of incantations. O, Gula keep me healthy and accept your fee. Incantation spell. ${ }^{108}$ 'It is an invocation for a sick eye.

Overview of IGI 1 Manuscripts and Parallels: AA=BAM 20; AB=BAM 159; AC=BAM 19; AD=BAM 22; AE=BAM 18; AmA=BAM 165; AX=KAL 7/12; bA=BM 41293+; EA=EMAR 6/4; HA=STT 279; NA=BAM 510; NB=BAM 513; NC=BAM 514; ND=BAM 515; NE=BAM 516; NJ=AMT 12/5; NQ=BAM 521; NU=AMT 14/3; sA=IRAQ 65; UA=AuOrS 23/27; uA=SpTU 50; UB=Ugaritica 5; UC=AuOrS 23/14; xA=CM 37 
NB iii 2 [ÉN igi bar igi b]ar-bar igi bar-ra bar-bar igi huš igi huš igi bar-ra huš-huš

NC iii begins.

(UC) r.10 TU6.ÉN.É.[NU.RU ..........................] igi huš igi huš-huš igi bar-ra huš-huš

99' NA ii 36' $a-p] a-t u ́$ IGI.MIN $a$-šá-tú IGI.MIN ša MU[D ..]

NB iii 3 [igi bar ná-a igi] bar da-a igi bar hul-a IGI.MIN $a$-ba- $a$-tu 'IGI.MIN? $\ulcorner a\urcorner$-ša-tu IGI.MIN ša MUD DIRI- $a$

100’ NA ii 37'

NB iii 4 $q a \hat{a}-b] u-u ́$ šá ${ }^{\mathrm{d}} g u-{ }^{\mathrm{r}} l a^{\urcorner}$

(AX) ii' 1' ana- ${ }^{\circ} k u^{? ?} \mathrm{x}[$.<smiles>[131IH]</smiles>
......................................] x 50 NA 50 sa-niq qá-bu-ú šá d gu-la

101' NA ii 38'

[.. p]a-rik KUR-ú

NB iii 5

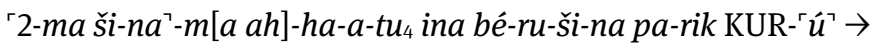

102' NA ii 39'

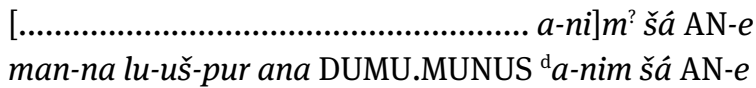
NB iii 5 (AX) ii' 2' man-na [

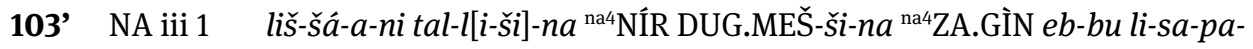
$a-n i$ A.MEŠ A.AB.BA

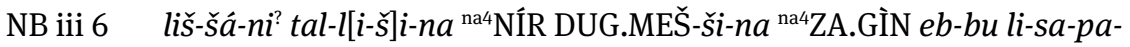
$a-n i$ A.MEŠ A.AB.BA $\rightarrow$

(AX) ii’ 3ff.' liš-šấ-' $a^{\urcorner}-[n i$.....................] / kar-p[a-ti-ši-na ] / li-s[a?-pa$a-n i$ . $/ i-n[u$ ..]

(EA) 11 li-ši-a-ni ú-ša-ba-ni me-e A.BA DAGAL-ti

104’ NA iii 2 tam-tì DAGAL-te ša 'ha-riš’-tu la ú-ri-du ana lìb-bi mu-suk-ka-tú la LUH-u qa-ti-šá

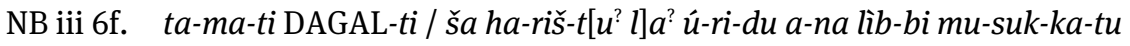
la LUH-u qa-te-šá

105’ NA iii 3 lis-la-ni-im-ma 'li’-ke-eș-șa-a KÚM și-ri-ih-tú šá ŠÀ IGI.MIN-šú NB iii 8 lim-la-ni-im-m[a? l]i-ke-eș-ṣa-a KÚM și-ri-ih-tu šá ŠÀ IGI.MIN-šú (AX) ii' 7' ana IGI.M[IN-šú

Overview of IGI 1 Manuscripts and Parallels: AA=BAM 20; AB=BAM 159; AC=BAM 19; AD=BAM 22; AE=BAM 18; AmA=BAM 165; AX=KAL 7/12; bA=BM 41293+; EA=EMAR 6/4; HA=STT 279; NA=BAM 510; NB=BAM 513; NC=BAM 514; ND=BAM 515; NE=BAM 516; NJ=AMT 12/5; NQ=BAM 521; NU=AMT 14/3; sA=IRAQ 65; UA=AuOrS 23/27; uA=SpTU 50; UB=Ugaritica 5; UC=AuOrS 23/14; xA=CM 37 
106' NA iii 4 ÉN ul ia-at-t[u]-un ÉN ${ }^{\mathrm{d}} 40 u^{\mathrm{d}}$ asal-lú-hi ÉN ${ }^{\mathrm{d}}$ da-mu u ${ }^{\mathrm{d}}$ gu-la NB iii 9 ÉN ul ia-a[t-tu-un É]N ${ }^{\mathrm{d}} 40 u^{\mathrm{d}}$ asal-lú-hi ${ }^{\Gamma E N^{\mathrm{d}}} d a^{7}-m u u^{\mathrm{d}} g u-l a$ (AX) ii’ 8f.' É[N . $/$ rd $\urcorner[$

107’ NA iii 5 ÉN ${ }^{\mathrm{d}}$ nin- ${ }^{-}$girima ${ }^{7}$ be-let šip-te ${ }^{\mathrm{d}} g u$-la TI.LA-ma NÍG.BA-ki TI-i TU 6 .ÉN

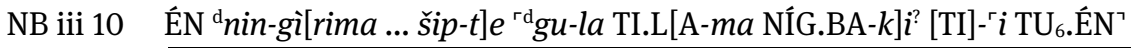

NC iii 10' [ÉN] ' ${ }^{\mathrm{d}}$ nin-girima be-let šip-te ${ }^{\mathrm{d}} \mathrm{gu}{ }^{\top}-[\mathrm{la}$............................................]

(AX) ii' 10’

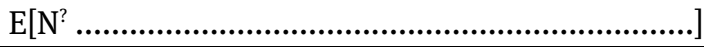

108’ NA iii 6 KA.INIM.MA IGI GIG.GA.A.KÁM

NB iii $11 \quad$ KA.INIM.MA [.................................]

NC iii 11' KA.INIM.MA [..................................]

(AX) ii’ 11f.' K[A.INIM.MA .......] / u x [......]

109’ dudubi annû ša šīpāti sāmāti țurra tațammi sebet kișrī takașșar ēma takașṣaru šipta tamannu ina īnī̌u marušti tarakkas
${ }^{109}$ 'Its medical application: you spin that which is a cord of red wool, you shall tie seven knots (on it). Whenever you make a knot, you shall recite the incantation (see above). You shall bind (it) on his sick eye!

109’ NA iii 7 DÙ.DÙ.BI $a n-n u-u$ ša [SÍG S]A ${ }_{5}$ DUR NU.NU 7 KA.KEŠDA KEŠDA $e$-ma 'KEŠDA ÉN' ŠID-nu ina IGI-šú GIG-tì KEŠDA

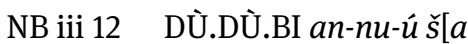

NC iii 12’ DÙ.DÙ.BI an-nu-u ša SÍG SA ${ }_{5}{ }^{\ulcorner D U R}$ ' NU.NU 7 KA.KE[ŠDA

(AX) ii’ 13’ D[Ù.DÙ.BI

Overview of IGI 1 Manuscripts and Parallels: AA=BAM 20; AB=BAM 159; AC=BAM 19; AD=BAM 22; AE=BAM 18; AmA=BAM 165; AX=KAL 7/12; bA=BM 41293+; EA=EMAR 6/4; HA=STT 279; NA=BAM 510; NB=BAM 513; NC=BAM 514; ND=BAM 515; NE=BAM 516; NJ=AMT 12/5; NQ=BAM 521; NU=AMT 14/3; SA=IRAQ 65; UA=AuOrS 23/27; uA=SpTU 50; UB=Ugaritica 5; UC=AuOrS 23/14; $\mathbf{x A = C M ~} 37$ 
110’ šiptu igi bar igi bar-bar igi bar-ra barbar igi huš igi huš-huš igi bar-ra hušhuš

111' igi bar ná-a igi bar da-a igi bar hul-a ìnà apâtu ìnā ašâtu

112' ìna pursìt dāmi šuteșlipātu ammīn tabbâ ammīn taššâ

113’ ammīn ikkalkināši bāṣu ša nāri taltallû ša gišimmari

114' ša titti niqqāša ša ari tibnī̌su alsīkināši alkāni

115’ ul alsīkināǒs ul tallakāni lām itbâkkināši šūtu iltānu šadû amurru šiptu

116’ ka'inimma īni marušti
${ }^{110}$ Incantation: The open eye is a staring eye, the opened eye stares, the reddish eye is a crimson eye, the opened eye is crimson. ${ }^{111}$ 'The open eye is drowsy, the open eye is weak, the open eye is harmed (lit. evil). $\mathrm{O}$ cloudy eyes, $\mathrm{O}$ blurred eyes, ${ }^{112} \mathrm{O}$ eyes intersected by blood vessels! Why are you cloudy? Why are you blurry? ${ }^{113}$ 'Why does the sand of the river, the pollen of the date palm ${ }^{114}$ '(or) the fig, (or) chaff of its straw ${ }^{113}$ 'cause you pain? ${ }^{114}$ 'I called to you, come here (to hide)! ${ }^{115}$ Did I not call you? Will you not come here, before the south, north, east (and) west wind rise up against you? (This is) the incantation. ${ }^{116}$ It is an invocation for a sick eye.

110' NA iii 8 ÉN igi bar ig[i ............. bar-r]a 'bar'-bar igi huš igi huš-'huš huš-huš

NB iii 13 ÉN igi bar igi bar-bar i[gi

NC iii 13’ ÉN igi bar igi bar-bar igi bar-ra bar-bar igi huš igi h[uš-hu]š 'igi bar`ra 'huš'-huš

(AX) i’ 1f.' bar]-bar / [ ..]-'huš ${ }^{\urcorner}$

(UC) r.10 TU6.ÉN.É.[NU.RU..............................] igi huš igi huš-huš igi bar-ra huš-huš

111’ NA iii 9 igi bar n[á-a .................] igi bar hul-a IGI.MIN $a$-pa-tu IGI.MIN $a$-ša-tu NB iii 14 igi-bar ná-a igi-ba[r

NC iii 14’ igi-bar ná-a igi-bar da-a igi-bar hul-a IGI.MIN ‘ $a-p a\urcorner-t u$ IGI.MIN $a$-ša${ }^{\ulcorner} t u{ }^{\top}$

(AX) i’3f.' [ h]ul-a / [ ] šá MÚD DIRI- $a$

112’ NA iii 10 IGI.MIN pur-[sít MÚD šu-te-eș-li-p] $a^{\ulcorner}{ }^{\ulcorner} a^{\urcorner}$-tu am-mìn tab-ba-a $\left.\left.{ }^{\ulcorner} a m-m i n\right\urcorner\right\urcorner$ 'taš-šá-a'

Overview of IGI 1 Manuscripts and Parallels: AA=BAM 20; AB=BAM 159; AC=BAM 19; AD=BAM 22; AE=BAM 18; AmA=BAM 165; AX=KAL 7/12; bA=BM 41293+; EA=EMAR 6/4; HA=STT 279; NA=BAM 510; NB=BAM 513; NC=BAM 514; ND=BAM 515; NE=BAM 516; NJ=AMT 12/5; NQ=BAM 521; NU=AMT 14/3; SA=IRAQ 65; UA=AuOrS 23/27; uA=SpTU 50; UB=Ugaritica 5; UC=AuOrS 23/14; xA=CM 37 
NB iii 15 'IGI.MIN pur-sít MÚD’ $t a b-b] a-\ulcorner a$ am-min taš-šá-a $a^{\urcorner}$

NC iii 15, IGI.MIN pur-sít MÚD šu-te-eș-li-pa-a-tu am-mìn tab-ba-a am-mìn taš-šá-a

(AX) i’ 5f.' šu-har]-ra-a-te / [ ]- ${ }^{-} n i^{?}$ taš $-\check{s} \check{a}-a$

113’ NA iii 11 am-mì-n[i $b a-a-s ̦] u$ ša $n[a-a-r i$

NB iii 16 t]al-tal-lu-ú šá giš GIŠIMMAR

NC iii 16’ am-mì-ni ik-kal-ki-na-ši ba-a-șu ša na-a-ri tal-tal-lu-ú šá gišr GIŠIMMAR $\urcorner$

(AX) i’ 7f.' .]--̌̌i ba-a-ṣu šá na-a-ri / [..

114’ NA iii 12 šá ti-[it-tu ni-iq-qa-š] $a[$

NB iii 17

$[.$. IN.NU-š]u al-si-ki-na al-ka-n[i]

NC iii 17’ šá ti-it-tu ni-iq-qa-šá šáa a-ri-i IN.NU-šu al-si-ki-na-ši al-ka-ni

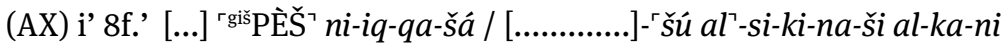

115’ NA iii 13 ul a[l-si-ki-na-ši ul ta-la]-k[a-ni ....]

NB iii 18 rul al-si’-[ki-na-ši

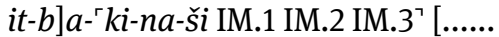
....]

NC iii 18’ ul al-si-ki-na-ši ul ta-la-ka-ni la-am it-ba-ki-na-ši IM.1 IM.2 IM.3 IM.4 'ÉN'

(AX) i' 10f.' [. .]- 'na-ši ul tal-la-ka-ni / [.

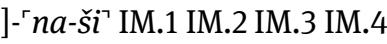
ÉN

116' NA iii 14 KA.[INIM.MA ...]

NB iii 19 KA.INIM.MA IGI GIG.GA.[.......]

NC iii 19’ KA.INIM.MA IGI GIG.GA. ' KÁM ${ }^{\prime}$

Overview of IGI 1 Manuscripts and Parallels: AA=BAM 20; AB=BAM 159; AC=BAM 19; AD=BAM 22; AE=BAM 18; AmA=BAM 165; AX=KAL 7/12; bA=BM 41293+; EA=EMAR 6/4; HA=STT 279; NA=BAM 510; NB=BAM 513; NC=BAM 514; ND=BAM 515; NE=BAM 516; NJ=AMT 12/5; NQ=BAM 521; NU=AMT 14/3; SA=IRAQ 65; UA=AuOrS 23/27; uA=SpTU 50; UB=Ugaritica 5; UC=AuOrS 23/14; $\mathbf{x A = C M ~} 37$ 
(AX) i’ 12.' 'IGI? GIG.GA.A.KÂM

\begin{tabular}{|c|c|c|}
\hline \multicolumn{2}{|c|}{$\begin{array}{l}\text { 117’ dudubi šīpāti sāmāti šīpāti peșâti } \\
\text { ahennâ tațammi sebet u sebet kiṣrī } \\
\text { takașșar ēma takașșaru šipta } \\
\text { tamannu } \\
\text { 118’ țurri šīpāti sāmāti ina īnīšu marušti } \\
\text { tarakkas țurri šīpāti peșâti ina īnīšu } \\
\text { balitti tarakkasma ina'eš }\end{array}$} & $\begin{array}{l}{ }^{117} \text { 'Its medical application: you spin } \\
\text { separately red (and) white wool. You } \\
\text { shall tie seven and seven knots (on each } \\
\text { wool). Whenever you make a knot, you } \\
\text { shall recite the (above) incantation. } \\
{ }^{118} \text { 'You tie the cord from the red wool on } \\
\text { his sick eye. You tie the cord from the } \\
\text { white wool on his healthy eye and then } \\
\text { it (the eye) he should get better. }\end{array}$ \\
\hline & \multicolumn{2}{|c|}{ 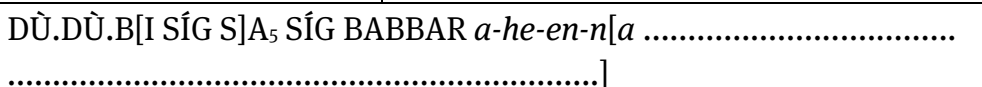 } \\
\hline & \multicolumn{2}{|c|}{$\begin{array}{l}\text { DÙ.DÙ.BI SÍG SA }{ }^{r}{ }^{r} \text { IÍG }^{\urcorner} \mathrm{B}[\mathrm{ABBAR} \\
\text { KEŠDA } e \text {-ma KEŠDA ÉN Š[ID-nu] }\end{array}$} \\
\hline & \multicolumn{2}{|c|}{$\begin{array}{l}\text { DÙ.DÙ.BI SÍG SA }{ }_{5} \text { SÍG BABBAR } a \text {-he-en-na- } a \text { NU.NU } 7 \text { u } 7 \text { KA.KEŠDA } \\
\text { KEŠDA } e \text { - } m a{ }^{\ulcorner} \text {KEŠDA ÉN ŠID }{ }^{-} n[u]\end{array}$} \\
\hline & \multicolumn{2}{|c|}{ [.............................. SÍ] B BABBAR ${ }^{\ulcorner} a-h e^{\urcorner}-n a-a$ NU.NU 7 u 7} \\
\hline
\end{tabular}

118’ NA iii 16 DUR S[ÍG] ' $\mathrm{SA}_{5}{ }^{\urcorner}$ina IGI-šú GIG-tì [

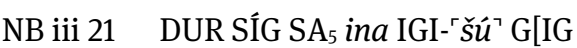
D]UR SÍG BABBAR ina IGI-šú TI.LA KEŠDA-ma i[na-eš]

NC iii 21’ 'DUR SÍG SA ${ }^{\urcorner}$ina IGI-šú GIG-tì KEŠDA DUR SÍG BABBAR

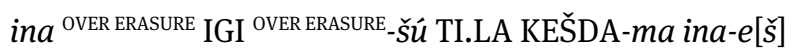

(AX) i’ 14f.'DUR SÍG SA ${ }_{5}{ }^{\prime}$ ina IGI-šú GIG KEŠDA-`á $s^{\urcorner}$/ [ ina IG]I- '̌̌ú $b a\urcorner$-lit-ti KEŠDA-ma T[I.LA]

119’ šiptu igi bar igi bar-bar igi bar-ra barbar igi hul igi hul-hul igi bar-ra hulhul

120’ šittāšina mārat Ani ina berūšina pitiqtu patqat

\begin{abstract}
${ }^{119}$ 'Incantation: The open eye is a staring eye, the opened eye stares, the evil eye is malevolent, the opened eye is malevolent! ${ }^{120}$ The two of them are daughter(s) of Anu, between whom (the eyes) a mud-brick wall is constructed
\end{abstract}

Overview of IGI 1 Manuscripts and Parallels: AA=BAM 20; AB=BAM 159; AC=BAM 19; AD=BAM 22; AE=BAM 18; AmA=BAM 165; AX=KAL 7/12; bA=BM 41293+; EA=EMAR 6/4; HA=STT 279; NA=BAM 510; NB=BAM 513; NC=BAM 514; ND=BAM 515; NE=BAM 516; NJ=AMT 12/5; NQ=BAM 521; NU=AMT 14/3; sA=IRAQ 65; UA=AuOrS 23/27; uA=SpTU 50; UB=Ugaritica 5; UC=AuOrS 23/14; xA=CM 37 


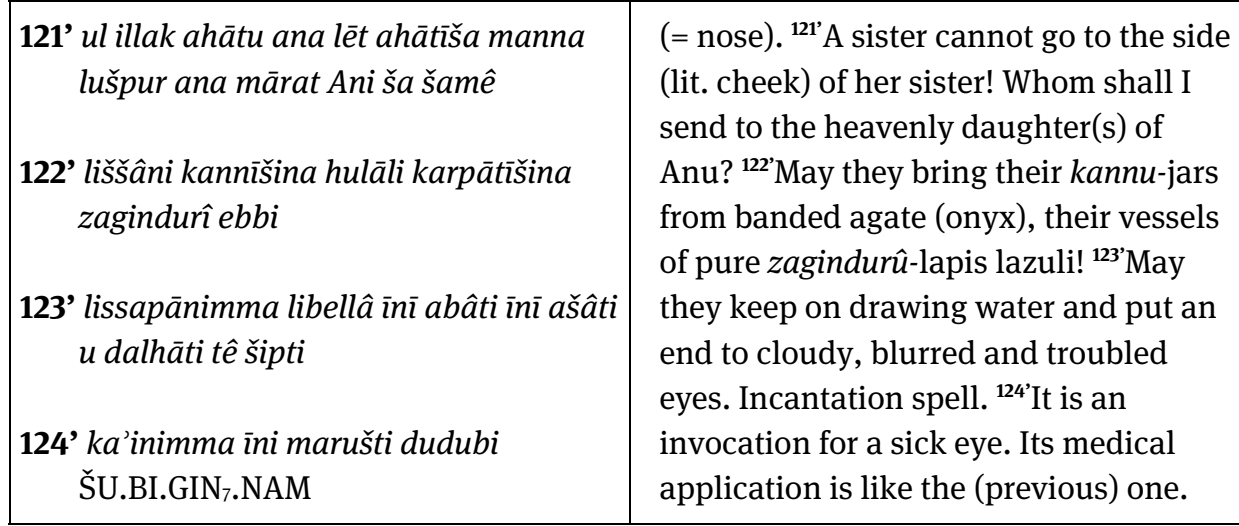

119' NA iii 17 ÉN ig[i bar] 'igi` bar-bar igi bar-ra `bar`-ba[r ........ h]ul-`hul igi` ba[rra .............]

NB iii 22 ÉN igi bar igi bar-bar igi bar-r[a bar-bar igi h]ul igi hul-hul igi bar-ra hul-h[ul]

NC iii 22’ 'ÉN igi bar igi` bar-bar igi bar-ra bar-bar igi hul igi `hul??-hul igi barra 'hul'-h[ul]
(AX) i' 16f.'[ bar?-ra? hul? - $-[$ hul] hull rigi?

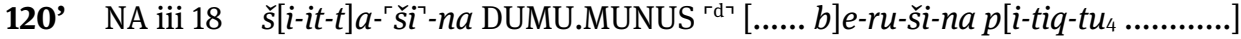

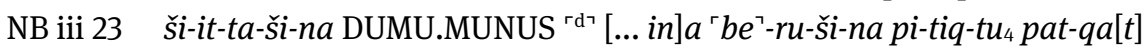
NC iii 23' [ši-it]-'ta-ši-na DUMU.MUNUS ' ${ }^{\text {a } a-n i ~ i n a ~ b e-r u-s ̌ i-n a ~ p i-t i q-t u ~}{ }_{4}$ pat-[qat]

121' NA iii $19 \quad u\left[l^{?}\right.$.............................a-ha-ti-š $] \hat{a}^{?}$ man-na lu-uš-pur $\left.{ }^{\ulcorner} a\right\urcorner-[$ na (or $a[$ na)

NB iii 24 ul il-lak a-ha-tu a-n[a le-e]t ${ }^{\ulcorner} a^{\urcorner}$-ha-ti-šá man-na lu-uš-pur a-na DUMU.MUNUS ${ }^{\mathrm{d}} a-n i \grave{s} \check{s}^{\mathrm{r}} \mathrm{AN}-e^{\urcorner}$

NC iii 24’ ul i[l-la]k'a-ha-tuq ${ }^{?\urcorner}$ ana le-et a-ha-ti-šá man-na lu-uš-pur ana 'DUMU'.MUNUS ${ }^{\mathrm{d}} a$-nì šá AN-[e]

(AX) ii’ 2'

man-na [lušpur ..............]

122’ NA iii 20 D]UG.MEŠ-ši-na ${ }^{\mathrm{na} 4}[$

NB iii 25 liš-šá-ni kan-ni-ši-na ${ }^{\text {na4 }}$ N[ÍR] DUG.MEŠ-ši-na ${ }^{\text {na4ZA.GÌN.DURU }}{ }_{5} e b-b u$ NC iii 25, liš-šá-a-n[i kan-n]i-ši-na ${ }^{\text {na4NÍR DUG.MEŠ-ši-na DUG ZA.GÌN.DURU }}$ $e b-b[u]$

Overview of IGI 1 Manuscripts and Parallels: AA=BAM 20; AB=BAM 159; AC=BAM 19; AD=BAM 22; AE=BAM 18; AmA=BAM 165; AX=KAL 7/12; bA=BM 41293+; EA=EMAR 6/4; HA=STT 279; NA=BAM 510; NB=BAM 513; NC=BAM 514; ND=BAM 515; NE=BAM 516; NJ=AMT 12/5; $\mathbf{N Q = B A M ~ 5 2 1 ; ~ N U = A M T ~ 1 4 / 3 ; ~ S A = I R A Q ~ 6 5 ; ~ U A = A u O r S ~ 2 3 / 2 7 ; ~} \mathbf{u A}=$ SpTU 50; UB=Ugaritica 5; UC=AuOrS 23/14; $\mathbf{x A = C M ~} 37$ 
(AX) ii’ 3f.' liš-šá $\left.{ }^{-} a\right\urcorner-[n i$ ] / kar-pa-[ti-ši-na

123’ NA iii 21

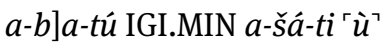

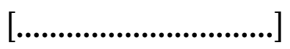

NB iii 26f. li-is-sa-pa-ni-im-m[a] li-be-la-a IGI.MIN a-ba-ti / IGI.MIN $a$-šá- ${ }^{\ulcorner} t i \grave{u}{ }^{\urcorner}$ dal-ha-a-ti ${ }^{\top} \mathrm{TU}_{6}{ }^{\urcorner} . \mathrm{E} \mathrm{N}$

NC iii 26’ li-is-sa-'pa`-ni-im-ma li-be-la-a IGI.MIN a-ba-tú IGI.MIN $a$-šáa-ti ù dal-ha-a-ti T[U6.ÉN]

(AX) ii' 5f.' li-s[a'-pa-a-ni .] $/ i-n[u$ ..]

124' NA iii 22f. ] IGI GIG.[GA .] ŠU.BI.[GIN $7 . N A M]$

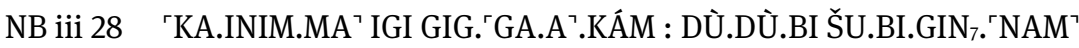

NC iii 27’ KA.INIM.MA `IGI’ GIG.GA.KÁM : DÙ.DÙ.BI ŠU.'BI’.GI[N7.NAM]

\begin{tabular}{|c|c|}
\hline $\begin{array}{l}\text { 125’ šiptu igi bar igi bar-bar igi bar-ra bar- } \\
\text { bar igi sùh igi sùh-sùh igi bar-ra sùh- } \\
\text { sùh } \\
\text { 126’ šitta īnū ahhātu šinama ina berūšina } \\
\text { šadû parik [ma] } \\
\text { 127' elēnūšina kișirtu kașrat šaplānūšina } \\
\text { pitiqtu patqat } \\
\text { 128’ ayyu šāršinama ayyu lā šāršinama }\end{array}$ & $\begin{array}{l}{ }^{125} \text { 'Incantation: The open eye is a staring } \\
\text { eye, the opened eye stares, the blurred } \\
\text { eye blurs vision, the opened eye is } \\
\text { blurred! }{ }^{1{ }^{\prime 2}} \text { 'Two eyes, both are sisters, } \\
\text { between whom a mountain is parked! } \\
{ }^{127} \text { 'Above whom the 'brow' is furrowed, } \\
\text { below whom a mud-brick wall (= nose) } \\
\text { is constructed! }{ }^{128} \text { 'Which one is their } \\
\text { (toxic) wind, which one is not their } \\
\text { (toxic) wind? }{ }^{129} \text { Which is the wind rising } \\
\text { against them, which is not a wind, rising } \\
\text { against them? }{ }^{130} \text { 'You, Marduk, disperse } \\
\text { the resistance, gloom, and anger! } \\
\text { [Incantation spell.] }{ }^{131} \text { 'It is an invocation } \\
\text { for a sick eye. Its medical application is } \\
\text { like the (previous) one. }\end{array}$ \\
\hline
\end{tabular}

NB iii 29 ÉN igi bar igi bar-bar igi bar-ra bar-`bar` igi sùh igi sùh-sùh igi bar-ra sùh-sù[h]

Overview of IGI 1 Manuscripts and Parallels: AA=BAM 20; AB=BAM 159; AC=BAM 19; AD=BAM 22; AE=BAM 18; AmA=BAM 165; AX=KAL 7/12; bA=BM 41293+; EA=EMAR 6/4; HA=STT 279; NA=BAM 510; NB=BAM 513; NC=BAM 514; ND=BAM 515; NE=BAM 516; NJ=AMT 12/5; NQ=BAM 521; NU=AMT 14/3; SA=IRAQ 65; UA=AuOrS 23/27; uA=SpTU 50; UB=Ugaritica 5; UC=AuOrS 23/14; xA=CM 37 
NC iii 28' ÉN igi bar igi bar-'bar igi bar`-ra bar-bar igi sùh igi sùh-sùh i[gi ba]r?'ra sùh ${ }^{\urcorner} s[u ̀ h]$

126’ NA iii 25 in] a be-ru-ši-na KUR-'̌́' [...........]

NB iii 30 ši-it-ta i-nu a-ha-tu ši-[na]-ma ina be-ru-ši-na KUR-ú pa-ri[k-ma]

NC iii 29' ši-it-ta i-nu a-'ha-tu' ši-na-ma ina be-r[u-š]i-na KUR-ú pa-r[ik-ma]

127' NA iii 26

[........................................ K]I.'TA ${ }^{\urcorner}-n u$-ši-na pi-ti[q-tú ............]

NB iii 31

NC iii 30' UGU-nu-ši-na ki-rsir-tu kàs-rat KI TA '-nu-ši-na pi-tiq-tu [

128' NA iii 27

$a-a]-{ }^{r} u^{\urcorner} \mathrm{NU} \mathrm{I}[\mathrm{M}-s ̌ i-n a-m a]$

NB iii $32 a-a-u ́$ IM-ši-na-a-ma $a-a-u ́$ NU IM- $\ulcorner\check{s} i-n[a-m a]$

NC iii 31’ $a-a-u ́$ IM-ši-na-'ma $a-a^{\urcorner}-u ́$ NU IM-ši-n[a?-ma]

129' NA iii 28

$l a]-\ulcorner a\urcorner \mathrm{I}[\mathrm{M}$ ti-bi-ši-na (-ma?)]

NB iii $33 a-a-u ́$ IM ti-bi-ši-na $a-a-u ́$ la-a IM ti-b[i-ši-na (-ma? $)]$

NC iii 32’ a-a-ú IM ti-bi-ši-'na' a-a-ú NU IM ti-bi-ši-n[a? (-ma? $)]$

130' NA iii 29 break of $\mathbf{K} 11847$

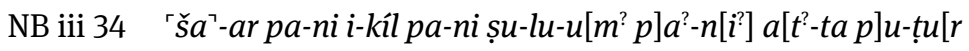

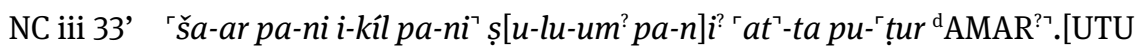
TU6.ÉN]

131' NB iii 35 [KA.INIM.M]A IGI GIG.GA.A.K[ÂM ......

NC iii 34' [KA.IN]IM.[MA IGI GIG.GA.KÂM : DÙ].DÙ.B[I] ŠU.'BI'.GI[N

132’ šiptu [igi bar] igi bar-bar [...] igi bar huš-huš

133' [...] igi bar-ra nu gi-na

134' ... [... sāniq] qabû ša Anu tê šipti

135’ ka'inimma ìni marušti : dudubi

ŠU.BI.GIN7.NAM
${ }^{132}$ Incantation: the open eye is a staring eye [...] the open eye is crimson. ${ }^{133}$ '.... the opened eye is not in order. ${ }^{134}$ '... [... the one who executes] the command of Anu. Incantation spell. ${ }^{135}$ It is an invocation for a sick eye. Its medical application is like the (previous) one.

Overview of IGI 1 Manuscripts and Parallels: AA=BAM 20; AB=BAM 159; AC=BAM 19; AD=BAM 22; AE=BAM 18; AmA=BAM 165; AX=KAL 7/12; bA=BM 41293+; EA=EMAR 6/4; HA=STT 279; NA=BAM 510; NB=BAM 513; NC=BAM 514; ND=BAM 515; NE=BAM 516; NJ=AMT 12/5; $\mathbf{N Q = B A M ~ 5 2 1 ; ~ N U = A M T ~ 1 4 / 3 ; ~ S A = I R A Q ~ 6 5 ; ~ U A = A u O r S ~ 2 3 / 2 7 ; ~} \mathbf{u A}=$ SpTU 50; UB=Ugaritica 5; UC=AuOrS 23/14; $\mathbf{x A = C M ~} 37$ 
132' NB iii 36 ig]i 'bar-bar` x [.

NC iii 35’ É[N igi bar i]g[i bar-bar i]gi bar huš-huš

133’ NC iii 36’ .] igi bar-ra nu gi-na

134' NC iii 37’ $\mathrm{x}$ [ $s a-n i q q] a ́ a-b u-u$ šá ${ }^{\mathrm{d}} a-n i \mathrm{TU}_{6}$.ÉN

135’ NC iii 38’ [KA.INIM.MA IGI GIG.GA.KÁM : DÙ.DÙ.B]I ŠU.'BI'.GIN7.NAM

\begin{tabular}{|c|c|}
\hline 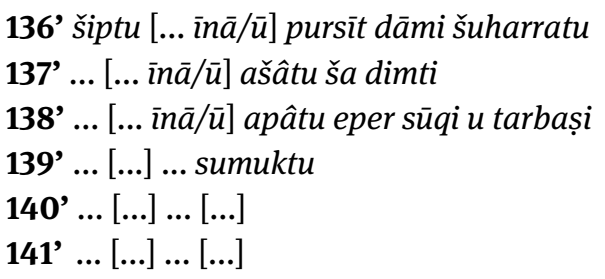 & 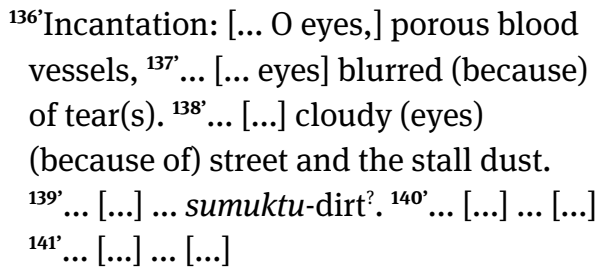 \\
\hline
\end{tabular}

136' NC iii 39' 'ÉN' $\left[\right.$ [..................................... IGI.MIN pur-s]i-'it MÚD šu-har-ra-tu ${ }^{\urcorner}$

(NA) ii 28' [....................šu-h]ar-ra-tú IGI.MIN ${ }^{\text {ERASURE }} \mathrm{GIN}_{7}$ nik-si UDU.NÍTA MÚD [.............]

(NB) ii 43’ [.......................... šu-h]ar-ra-te IGI.MIN GIN $n$ nik-si UDU. 'NÍTA MÚD še-en- $a^{\urcorner}$

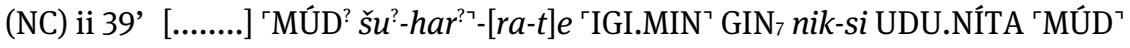
š $[e-e n-a]$

(UB) 4f. IGI.MEŠ pur-sí-it 'MÚD'.ME[Š] / šu-har-re-tu

(EA) 3 pur-si-it da-mi i-na-š[u]

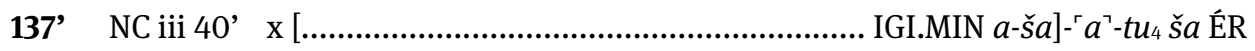

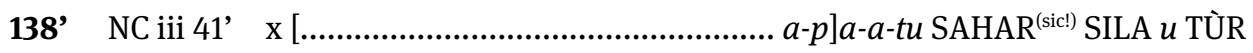

139' NC iii 42’ x [................................................................. x x su-muk-t[ú]

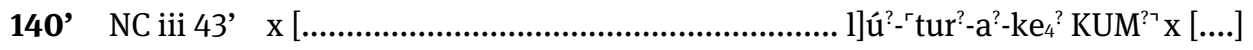

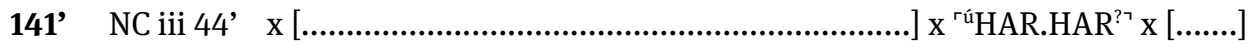

\section{GAP!}

Overview of IGI 1 Manuscripts and Parallels: AA=BAM 20; AB=BAM 159; AC=BAM 19; AD=BAM 22; AE=BAM 18; AmA=BAM 165; AX=KAL 7/12; bA=BM 41293+; EA=EMAR 6/4; HA=STT 279; NA=BAM 510; NB=BAM 513; NC=BAM 514; ND=BAM 515; NE=BAM 516; NJ=AMT 12/5; NQ=BAM 521; NU=AMT 14/3; sA=IRAQ 65; UA=AuOrS 23/27; uA=SpTU 50; UB=Ugaritica 5; UC=AuOrS 23/14; xA=CM 37 
The space on NB, between 79-7-8, 156 [AMT 8/3] and $\mathrm{K} \mathrm{7105,} \mathrm{is} \mathrm{unclear.} \mathrm{But} \mathrm{there} \mathrm{are} \mathrm{at}$ least ca. 11 lines missing on NB.

142’ NB iii 47a' .] $x$

143’ [dudubi ... šīpāti sāmāti šìpāti peșâti] ahennâ tatammi

144' [sebet kiṣrī takașșar ēma takașșaru šipta tamannu] ina nakkaptišu tarakkassu
143'[Its medical application:] you spin separately [red (and) white wool? ...] ${ }^{144}$ [You shall tie seven knots. Whenever you make a knot you shall recite the incantation.] You bind it on his temple.

143’ NB iii 47b’ [DÙ.DÙ.BI ] $a$-he-en-na- $a^{\urcorner}[\mathrm{N}] \mathrm{U} . \mathrm{NU}$

144' NB iii 47c' ] ina SAG.KI-šú KEŠDA- ${ }^{\ulcorner} s u^{\urcorner}$

\begin{tabular}{|l|l}
\hline 145’ [šiptu ...] ini lā iraššâ tê šipti & $\begin{array}{l}\text { 145' [Incantation: ... malady of] the eye } \\
\text { must not develop. Incantation spell. }\end{array}$
\end{tabular}

145' NB iii 47d' [ÉN .......................................................] x? IGI NU TUK- $a$ TU $_{6}$.ÉN

\begin{tabular}{|l|l|}
\hline 146' [dudubi ... ina nakkaptǐšu ša imitti & $\begin{array}{c}\mathbf{1 4 6}^{\prime} \text { [Its medical application: ... you bind it } \\
\text { on his'] right [temple? }\end{array}$
\end{tabular}

146’ NB iii 47e' [dudubi..... ina SAG.KI-šú? šá ZAG

147’ [šiptu ... ta-ma-ad-r]a-áš ta-ma-ad-ra- $\quad$ 147’[Incantation: ...] tamadraš tamadraš. áš tu -én $^{-e ́}$ Incantation spell.

147’ NB iii 47f' [ÉN ..................................... ta-ma-ad-r]a-áš ta-ma-ad-ra-áš tu

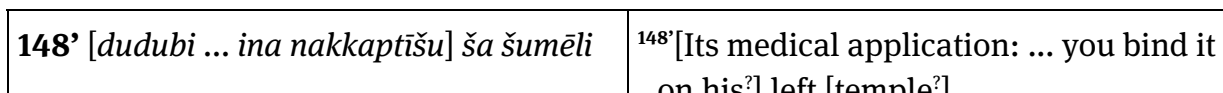
on his?] left [temple?].

148’ NB iii 47g’ [DÙ.DÙ.BI ................................................. ina SAG.KI-šú? šá GÙB

Overview of IGI 1 Manuscripts and Parallels: AA=BAM 20; AB=BAM 159; AC=BAM 19; AD=BAM 22; AE=BAM 18; AmA=BAM 165; AX=KAL 7/12; bA=BM 41293+; EA=EMAR 6/4; HA=STT 279; NA=BAM 510; NB=BAM 513; NC=BAM 514; ND=BAM 515; NE=BAM 516; NJ=AMT 12/5; NQ=BAM 521; NU=AMT 14/3; sA=IRAQ 65; UA=AuOrS 23/27; uA=SpTU 50; UB=Ugaritica 5; UC=AuOrS 23/14; xA=CM 37 
Begin of K 13465 on ms. NA

\begin{tabular}{|c|c|}
\hline $\begin{array}{l}\text { 149' [ka'inimma īni marušti }] \text { dudubi } \\
\text { ŠU.BI.GIN }{ }^{\text {S.NAM }}\end{array}$ & $\begin{array}{l}{ }^{149}[\text { It is an invocation for a sick eye.] Its } \\
\text { medical application is like the (previous) } \\
\text { one. }\end{array}$ \\
\hline \multicolumn{2}{|l|}{ 149' } \\
\hline \multicolumn{2}{|l|}{ NB iii 47h' [.... } \\
\hline 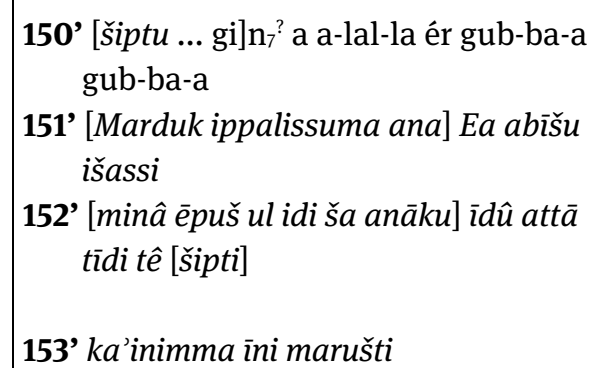 & $\begin{array}{l}{ }^{150} \text { 'Incantation: ... (pouring out)] like the } \\
\text { water of the shaduf, o tear(s) be present, } \\
\text { be present! }{ }^{151} \text { [Marduk noticed it (the } \\
\text { harm) and then] cried out [to] his father } \\
\text { Ea: }{ }^{152} \text { [what I must do I do not know: } \\
\text { what I] know, you (already) know. } \\
\text { Incantation spell. }{ }^{153} \text { '[It is an invocation] } \\
\text { for a sick eye. }\end{array}$ \\
\hline
\end{tabular}

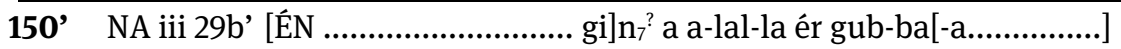

NB iii 47i' [ÉN ................................................. g]ub-ba-a gub-b[a-a]

151' NA iii 29c' [Marduk ip-pa-lis-su-ma ana $]^{\mathrm{rd}\urcorner} e^{\prime}-a^{\ulcorner} \mathrm{AD}^{\urcorner}-$šú $i-s ̌ a ́-a[s-s i]^{\prime}$

NB iii 47j' [..............................................

152’ NA iii 29d' [mi-na-a e-pu-uš ul i-di ša ana-ku i-du]-`u ?` at-ta ti-di T[U ${ }_{6}$.ÉN]

NB iii 47k' [....................................................... ' $t i-d i\urcorner \mathrm{T}\left[\mathrm{U}_{6} . \mathrm{EN}\right]$

153’ NA iii 29e' [ka’inimma IG]I GIG.GA.[KÁM]

154' dudubi šīpāti sāmāti šīpāti peșâti

ištēniš tațammi sebet u sebet kiṣrī

takașșar ēma takașșaru šipta

tamannu ina nakkapātǐ̌̌u

tarakkasma ina'eš
${ }^{154}$ Its medical application: you spin together red (and) white wool. Seven and seven knots you shall tie. Whenever you make a knot you shall recite the incantation. You bind it on his temples and he will get better.

Overview of IGI 1 Manuscripts and Parallels: AA=BAM 20; AB=BAM 159; AC=BAM 19; AD=BAM 22; AE=BAM 18; AmA=BAM 165; AX=KAL 7/12; bA=BM 41293+; EA=EMAR 6/4; HA=STT 279; NA=BAM 510; NB=BAM 513; NC=BAM 514; ND=BAM 515; NE=BAM 516; NJ=AMT 12/5; NQ=BAM 521; NU=AMT 14/3; sA=IRAQ 65; UA=AuOrS 23/27; uA=SpTU 50; UB=Ugaritica 5; UC=AuOrS 23/14; xA=CM 37 
154' NA iii 29f'

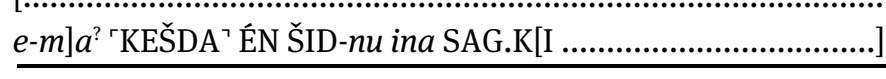

NB iv 1f.

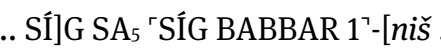
.. 1 ] KEŠDA ÉN `ŠID-nu? $i[n a$.

NC iv 1 'DÙ'.D[Ù.BI ..] '1-niš NU.NU 7 u 7 KA.KEŠDA KEŠDA e-ma KEŠDA? ÉN ŠID-nu ina SAG.KI.MEŠ-šú KEŠDA-ma ina-eš ${ }^{7}$

155’ [šiptu i-gi t]i-la a-ga ti-la i-gi kún-na a-ga kún-na : ur-sag gi-na-zu gi bànda-zu tu 6 -én

156’ ka'inimma īni marušti : dudubi ŠU.BI.GIN $7 . N A M$ 155' [Incantation: The eye] is healthy, the back is healthy, the eye is shining, the back is shining! Your tiny reed is your faithful hero. Incantation spell. ${ }^{156}$ It is an invocation for a sick eye. Its medical application is like the (previous) one.

155' NA iii $29 g-h^{\prime}$ ' n]a a-ga k[ún?-na

/..............z] $u^{?} \mathrm{tu}_{6}$-[én]

NB iv 3 [ÉN i-gi t]i-la a-ga ti-la i-gi kún-na `a?-[ga] 'kún`-n[a

NC iv 2 ti-l]a `i-gi’ kún-na a-ga kún-`na : ur-sag gi’-na-zu gi `bàn-da-zu tu -én $^{\urcorner}$ $. . \check{S}] U . B I . G I\left[N_{7 . N A M]}\right.$

NB iv 4 [KA.IN]IM.MA IGI GIG.GA.A.KÁ[M (:)] DÙ.DÙ. 'BI [ [.

$\mathrm{NC}$ iv 3 K[A.INIM.MA IGI] GIG.GA.A.KÁM : DÙ.DÙ.BI ŠU.BI.GIN

157’ šiptu pa-la huš pa-la huš pa-la huš-bi níg-gi-na-bi i-gi pa-la huš-bi tu -én $^{-e}$

158' ka'inimma ìni marušti dudubi ŠU.BI.AŠ.ÀM
157'Incantation: reddish 'robe', reddish 'robe', its 'robe' is reddish! In truth: the eye's 'robe' is reddish. Incantation spell. ${ }^{158}$ 'It is an invocation for a sick eye. Its medical application is the same (as the previous one).

157’ NA iii 29j' Presumably two lines until the beginning of col. iv.

Overview of IGI 1 Manuscripts and Parallels: AA=BAM 20; AB=BAM 159; AC=BAM 19; AD=BAM 22; AE=BAM 18; AmA=BAM 165; AX=KAL 7/12; bA=BM 41293+; EA=EMAR 6/4; HA=STT 279; NA=BAM 510; NB=BAM 513; NC=BAM 514; ND=BAM 515; NE=BAM 516; NJ=AMT 12/5; NQ=BAM 521; NU=AMT 14/3; SA=IRAQ 65; UA=AuOrS 23/27; uA=SpTU 50; UB=Ugaritica 5; UC=AuOrS 23/14; xA=CM 37 
NB iv 5 [ÉN pa-l]a 'huš` pa-la huš pa-la huš-'bi` ní[g-gi-n]a-bi i-gi pa-la rhuš ${ }^{-b}\left[i_{\text {tu }}\right.$-én]

NC iv 4 'ÉN pa-la huš pa-la huš pa-la huš-bi níg-gi-na-bi i-gi-i pa-la huš-bi tu ${ }_{6}$-én $\urcorner$

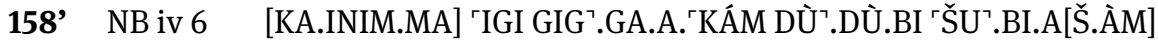

NC iv 5 'KA.INIM.MA IGI GIG.GA.A.KÁM` : DÙ.DÙ.BI ŠU.BI.AŠ.ÀM

159’ šiptu ìni ețli marșat ìni ardati marșat ini ețli $u$ ardati mannu uballit

160' tašappar ileqqûnikku libbi elleti gišimmari ina pîka teheppi ina qātīka tepettil

161' eṭla u ardata ina nakkapātǐšunu tukașșar in ețli u ardati iballuṭ tê šipti

162' ka'inimma ìni marušti dudubi ŠU.BI.AŠ.ÀM
${ }^{159}$ 'Incantation: The eye of the lad is sick; the eye of the maiden is sick! Who will heal the eye of the lad and the maiden? ${ }^{160}$ 'You will send for (and) they will bring you a clean date-palm offshoot! You break (it) up with your mouth (teeth) and twist (it to thread) with your hand. ${ }^{161}$ 'You bind (it) up on the lad's or maiden's temples and the eye of the lad and the maiden should recover. Incantation spell. ${ }^{162}$ It is an invocation for a sick eye. Its medical application is the same (as the previous one).

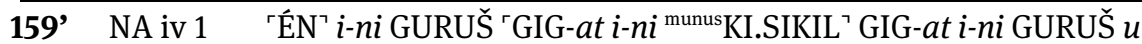
munusKI.SIKIL man-nu ú-bal-lit

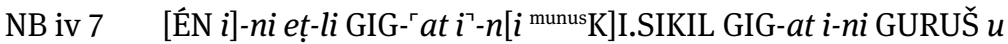
munusKI.SIKIL man-nu 'ú'-[bal-liț]

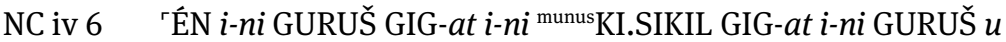
munusKI.SIKIL man-nu ú-bal'-liț

(HA) 17f. ÉN IGI GURUŠ GIG-ma IGI KI.SIKIL GIG-át / IGI GURUŠ $u$ KI.SIKIL man-nu ú-bal-laṭ

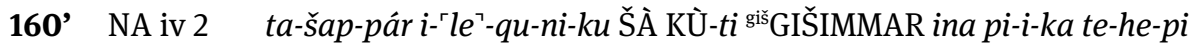
ina ŠU $k$ k te-pe-til

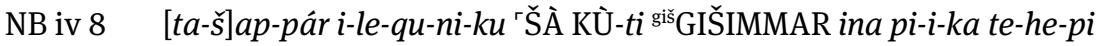
ina ŠU-ka te-pe - ti[l]

Overview of IGI 1 Manuscripts and Parallels: AA=BAM 20; AB=BAM 159; AC=BAM 19; AD=BAM 22; AE=BAM 18; AmA=BAM 165; AX=KAL 7/12; bA=BM 41293+; EA=EMAR 6/4; HA=STT 279; NA=BAM 510; NB=BAM 513; NC=BAM 514; ND=BAM 515; NE=BAM 516; NJ=AMT 12/5; NQ=BAM 521; NU=AMT 14/3; SA=IRAQ 65; UA=AuOrS 23/27; uA=SpTU 50; UB=Ugaritica 5; UC=AuOrS 23/14; xA=CM 37 


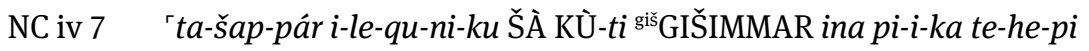
ina ŠU $-k a$ te-pe-til

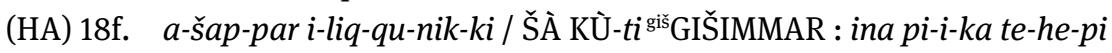
ina ŠU-ka SÚD

161’ NA iv 3 GURUŠ $u$ KI.SIKIL ‘ina SAG.KI.MEŠ ’-šú-nu tu-ka-șar IGI GURUŠ $u$ KI.SIKIL i-bal-lu-uṭ TU. ÉN

NB iv 9 [GURUŠ $u$ K]I.SIKIL ina SAG.KI.MEŠ-šú-nu tu-ka-șar IGI GURUŠ $u$

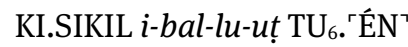

NC iv 8 'GURUŠ $u$ KI.SIKIL ina SAG.KI.MEŠ-šú-nu tu-ka-ṣar IGI GURUŠ $u$ KI.SIKIL $i$-bal-lu-ut TU $\mathrm{TU}_{6}$.ÉN ${ }^{\prime}$

(HA) 20f. IGI? (text BAD) GURUŠ u KI.SIKIL ina SAG.KI-šú tu? (text 'LI')-ka-șar / IGI GURUŠ $u$ KI.SIKIL $i$-bal-luṭ 'ÉN'

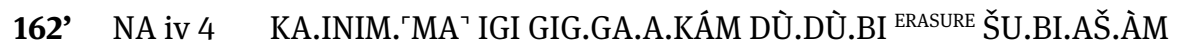

NB iv 10 [KA.I]NIM.MA IGI GIG.GA.A.KÁM DÙ.DÙ.BI ŠU.BI.AŠ.ÀM

NC iv 9 'KA.INIM.MA IGI GIG.GA.A.KÁM DÙ.DÙ.BI ŠU.BI.AŠ.ÀM

(HA) 22 KA.INIM IGI GI[G..........]

(HA) 23ff. DÙ.DÙ.BI SÍG S[A ${ }_{5}$ SÍG BABBAR 1-niš NU.NU] / 77 [u] 7 `KA.KEŠDA KE[ŠDA $e-m a$ KEŠDA] / 'ÉN? $\urcorner$ [Š]ID- $n\left[u\right.$......] / ${ }^{\text {r.1 }}$ ana SAG.KI.MIN-šú KEŠDA-m[a ina-eš/TIN]

\section{NB.: Billingual incantation, for the Sumerian see the score text (partiture) below!}

163’ šiptu ina šamê šāru izīqamma ina ìn amēli simmē ištakan

164' ištu šamê rūqūti

165' ana īnì marșāti simme ištakan

166’ ša amēlu šuātu ìnāšu dalhā

167’ īnāšu ašâ

168' amēlu šū ina ramānišu marșiš ibakki 169’ ša amēlu šuātu murussu Nammu imurma
${ }^{163}$ 'Incantation: The wind blew in the sky and thus caused lesions in a man's eye. ${ }^{164}$ From the distant sky, the wind blew in the sky and thus caused lesions in a man's eye. ${ }^{165}$ 'It caused lesions within the sick eyes! ${ }^{166^{\prime}}$ Regarding that man, his eyes are troubled, ${ }^{167}$ 'his eyes are blurred! ${ }^{168}$ 'This man weeps bitterly by himself. ${ }^{169}$ 'It was that man's illness

Overview of IGI 1 Manuscripts and Parallels: AA=BAM 20; AB=BAM 159; AC=BAM 19; AD=BAM 22; AE=BAM 18; AmA=BAM 165; AX=KAL 7/12; bA=BM 41293+; EA=EMAR 6/4; HA=STT 279; NA=BAM 510; NB=BAM 513; NC=BAM 514; ND=BAM 515; NE=BAM 516; NJ=AMT 12/5; NQ=BAM 521; NU=AMT 14/3; SA=IRAQ 65; UA=AuOrS 23/27; uA=SpTU 50; UB=Ugaritica 5; UC=AuOrS 23/14; xA=CM 37 
170' kasî hašlūti leqēma

171' šipat apsî idìma

172' inì amēli rukusma

173' Nammu ina qātīša elleti ìn amēli ina labātǐša

174' šāru ša ìn amēli uddupu ina īnī̌̌u littași

175’ ka'inimma īni marušti which the (water goddess) Nammu noticed (and said): ${ }^{170 ‘}$ Take crushed kasû-plant, ${ }^{171}$ 'recite the Apsû incantation, ${ }^{172}$ (and) bandage the eyes of the man.' ${ }^{173 '}$ 'When Nammu touches the man's eye with her clean hand, ${ }^{174}$ may the wind which has inflated the man's eye depart from his eye(s)! (Incantation!) ${ }^{175}$ 'It is an invocation for a sick eye.

163’ NA iv 5f. én an-na 'im? ri-a igi lú-ka gig-ga ba-an-gar / ina šá-me-e šâ-a-ru i-zi-qam-ma ina i-in LÚ si-im-me iš-ta-kan

NB iv 11f. [é]n an-na im ri-a igi lú-ka gig-ga ba-an-gar / ina šá-me-e šá- $\ulcorner a\urcorner-r u$ i-zi-qam-ma ina i-in LÚ si-im-me iš-ta-kan

NC iv 10f. 'én an-na im ri-a igi lú-ka gig-ga ba-an-gar / ina šá-me-e šá-a-ru i-zi-qam-ma ina i-in LÚ si-im-me iš-ta-kan

(HA) r.2ff. én an-na im 'ri-a’ igi 'lú'-k[a g]i[g-ga] / ba-an-gar : ina AN-e IM i-zi-qam-ma / ina i-ni LÚ sim-mu iš-ta-kan

164' NA iv 7f. an-ta 'sù'-da-ta im ri-a?(text MIN?) igi lú-ka gig-ga ba-an-gar / iš-tu AN-e ru-qu-ti

NB iv 13 'an?-ta sù-da-ta im ri-a igi lú-ka gig-ga ba-an-gar : iš-tu AN-e ru-qu-ti

NC iv 12f. 'an-ta sù-da-ta im ri-a igi lú-ka gig-ga ba-an-gar? / 'iš-tu AN-e $r[u]-$ $\left\ulcorner q u^{\urcorner}-t i\right.$

(HA) r.5ff. an-ta sù-ud-da im ri-a / igi lú gig ba-an-gar / ul-tú AN-e ru-qu-ti šá-aru / i-zi-qam-ma? ina i-ni LÚ sim-mu / iš-ta-kan

165' NA iv 9 igi gig-'ga' gig-ga ba-an-gar : ana i-ni mar-ṣa-a-ti si-im-me iš-ta-kan NB iv 14f. rigi gig’-ga gig-ga ba-an-gar / [...] 'i’-ni mar-șa-a-ti si-im-ma iš-ta-kan NC iv 14 rigi gig-ga gig-ga? [ . i-n] i m[ar-șa-a-ti si-i]m-me iš-ta-kan (HA) r.10 igi gig gig ba-an-gar

(HA) r.11 ina i-ni mar-șa-ti sim-mu iš-ta-kan

166’ NA iv 10 lú-bi igi-bi lù-lù-a : šá LÚ šu-a-tú i-na-šú da-al-ha NB iv 16f. [lú-b]i igi-bi lù-lù-a / [šá LÚ šu]-'a-tú i-da-šú da-'al'-h[a] NC iv 15 $i-n] a-s ̌ u ́ r d a-a l\urcorner-h a$

(HA) r.12 lú-bi igi-bi lù-lù-a

Overview of IGI 1 Manuscripts and Parallels: AA=BAM 20; AB=BAM 159; AC=BAM 19; AD=BAM 22; AE=BAM 18; AmA=BAM 165; AX=KAL 7/12; bA=BM 41293+; EA=EMAR 6/4; HA=STT 279; NA=BAM 510; NB=BAM 513; NC=BAM 514; ND=BAM 515; NE=BAM 516; NJ=AMT 12/5; NQ=BAM 521; NU=AMT 14/3; sA=IRAQ 65; UA=AuOrS 23/27; uA=SpTU 50; UB=Ugaritica 5; UC=AuOrS 23/14; xA=CM 37 
(HA) r.13 šá LÚ šu-u IGI.MIN-šú dal-ha

167’ NA iv 11 igi-bi 'ba'-an-`sùh'-sùh : i-na-šú $a$-šâa- $a$

NB iv 16f. 'igi-bi’ ba-an-'sùh'-sùh / [i-n]a-šśu $a$-šá-a

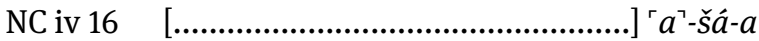

(HA) r.14 igi-bi sùh-sùh : i-na-šú $a$-šá-a

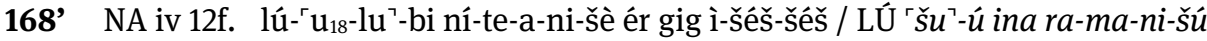
mar-și-iš i-bak-ki

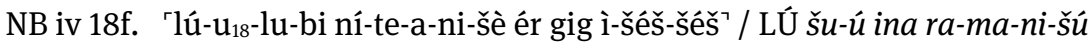
mar-și-iš $i-b[a k-k i]$

NC iv 17f. [lú-u $u_{18}$-lu-bi ní-te-a-ni-šè ér gig ì-š]éš-šéš / [ ..] 'i’-bak-ki

169’ NA iv 14 lú-'bi tu’-ra-a-ni ${ }^{\mathrm{d}}$ engur-ke ${ }_{4}$ igi `im-ma-an-sì : šá LÚ šú- $a$-tú mu-ru-us-su d MIN i-mur-ma

NB iv 20f. lú-bi tu-ra-a-ni dengur-ke ${ }_{4}$ 'igi im-ma?-a[n?-sì] / šá LÚ šú-a-tu $m u-r u-u s-s u{ }^{\mathrm{d}} \mathrm{MIN}$ 'i-mur-ma?

NC iv 19

$m u-r u-u s-s] u^{\mathrm{rd}} \mathrm{MIN} i-m u r^{\top}-m a$

(HA) r.15 lú-bi tu-ra-a-ni díd-ke 4 igi im-ma-an-sì

(HA) r.16 LÚ šú-a-tú GIG-su díD i-mur-ma

170' NA iv 15 gazi ${ }^{\text {rsarา }}$ gaz-gá šu ù-me-ti : ka-si-i haš-lu-ti le-qé-ma

NB iv 22f. gazi ${ }^{\mathrm{sar}}$ kum-mà 'šu’ ù-me-ti / ka-si-i haš-lu-ti le-qé-ma

NC iv $20 \quad$ [........................................................... le $q]$ é-ma

(HA) r.17 gazi ${ }^{\text {sar }}$ al-šeg ${ }_{6}$-gá šu u-me-ti

(HA) r.18 'ka-se-e ba?-áš-lu-te le-qé-ma

171’ NA iv $16{ }^{\ulcorner} \mathrm{tu}_{6}$ - $\mathrm{tu}_{6}{ }^{\top}$ abzu-ta u-me-ni-sì : ši-pat ap-si-i i-di-ma

$\mathrm{NB}$ iv 24f. tu $_{6}-\mathrm{tu}_{6}$ abzu-ta ù-me-ni-sì / ši-pat ap-si-i i-di-ma

$\mathrm{NC}$ iv 21 $i-d i]-m[a]$

(HA) r.19 [......... abzu-t]a u-m[e-ni-sì]

(HA) r.20 too broken, no dividing line visible, but expected.

172’ NA iv 17 i[gi l]ú-ka ù-me-ni-kešda : i-ni a-me-li ru-kus-ma

Overview of IGI 1 Manuscripts and Parallels: AA=BAM 20; AB=BAM 159; AC=BAM 19; AD=BAM 22; AE=BAM 18; AmA=BAM 165; AX=KAL 7/12; bA=BM 41293+; EA=EMAR 6/4; HA=STT 279; NA=BAM 510; NB=BAM 513; NC=BAM 514; ND=BAM 515; NE=BAM 516; NJ=AMT 12/5; NQ=BAM 521; NU=AMT 14/3; SA=IRAQ 65; UA=AuOrS 23/27; uA=SpTU 50; UB=Ugaritica 5; UC=AuOrS 23/14; $\mathbf{X A}=\mathrm{CM} 37$ 
NB iv 26f. igi lú-ka ù-me-ni-kešda / i-ni a-me-li ru-kus-ma NC iv 22 $r u-k u s-m] a^{?}$

(HA) r.21 [igi l]úu-'bi u-me-ni-kešda` : 'i-ni a-me ${ }^{\urcorner}-[l i$..........]

173’ NA iv 18f. [ ['engu]r lú šu kù-ga-na igi lú-ba-ka šu tag-ga-ni-ta / [ $\left.{ }^{\mathrm{d}} \mathrm{MI}\right] \mathrm{N}$ ina ŠU-šá KÙ-ti i-in a-me-lì ina la-ba-ti-šá

NB iv 28f. 'engur lú šu kù-ga-'na igi? l[ú-ba-ka šu tag-g]a-ni-ta / dMIN ina ŠU-š[á

$\mathrm{NC}$ iv 23f. too broken

(HA) r.22 díd šu kù-ga-a-ni igi lú-bi-ta šu t[ag-ga-ni-ta]

(HA) r.23 ${ }^{\mathrm{d}}$ MIN ina ŠU.MIN-šú KÙ-te i-ni LÚ ina la-pa-t[i-šú]

174' NA iv 20f. [im ig]i lú-ka sù-sù igi-bi-ta ba-ra-an-è / [ša-a]-ru šá i-in a-me-lì ud-du-pu ina i-ni-šú lit-ta-și

NB iv 30f. im igi lú-k[a ba-ra-a]n-è / šáa-a-rù šá ${ }^{\ulcorner} i^{\urcorner}-[i n$ lit-t]a-ṣi

NC iv 25f. ba-ra-a]n- '⿳亠̀⿵ / [ ..ina I]GI-'šú lit-ta-și

(HA) r.24 im igi lú-geš sù-sù igi-bi-šè ba-ra-'è

(HA) r.25 šá-a-ru šá i-ni LÚ ud-du-pu ina i-ni-šú lit-ta-și ÉN

175’ NA iv 22 [KA.I]NIM.MA IGI GIG.GA.A.KÁM

NB iv 32 'KA'.I[NIM.MA ..............].' 'A '.KÁM

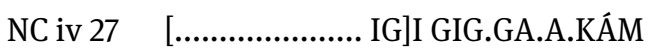

(HA) r.26 KA.INIM.MA šá IGI.MIN.MEŠ GIG.MEŠ

(HA) r.27f. DÙ.DÙ.BI GAZI ${ }^{\text {sar }}$ ŠEG 6 -šal SÚD / ÉN an-ni-te 7?-šú ana UGU ŠID-nu (HA) r.29 LAL-ma ina-ah

176' [šiptu] ìnū apâtu ìnū ašâtu ìnū pursindi dāmi šuharriātu 177’ šina ibakkâ ana pān ummišina Mami
${ }^{176}$ [Incantation.] O cloudy eyes, O blurred eyes, O porous blood vessels! ${ }^{177}$ The two (eyes) are weeping before their mother

Overview of IGI 1 Manuscripts and Parallels: AA=BAM 20; AB=BAM 159; AC=BAM 19; AD=BAM 22; AE=BAM 18; AmA=BAM 165; AX=KAL 7/12; bA=BM 41293+; EA=EMAR 6/4; HA=STT 279; NA=BAM 510; NB=BAM 513; NC=BAM 514; ND=BAM 515; NE=BAM 516; NJ=AMT 12/5; NQ=BAM 521; NU=AMT 14/3; sA=IRAQ 65; UA=AuOrS 23/27; uA=SpTU 50; UB=Ugaritica 5; UC=AuOrS 23/14; xA=CM 37 
178' ammīn nâšima ittīni tarkusī ašâ dāma u šăra tê šipti

179’ ka'inimma īni marušti
Mami: ${ }^{178 '}$ 'Why us and (why) did you saddle us with blurred (vision), blood, and wind?' Incantation spell. ${ }^{179}$ 'It is an invocation for a sick eye.

176' NA iv 23 [ÉN i-n] u a-pa-tú i-nu a-šá-tú i-nu pur-si-in-di da-a?(text MIN?)-mi šuhar-ri-a-tu

NB iv 33 šu-har-ri-a-t] $u_{4}$

NC iv 28 pur-si-in-d]i da-a-mi šu-har-ri-a-tu

177’ NA iv $24 \quad\left[\right.$ ši-n]a i-bak-ka-a ana IGI AMA-ši-na $\left.{ }^{\ulcorner d} m a-m i\right\urcorner$

NB iv 33 Broken

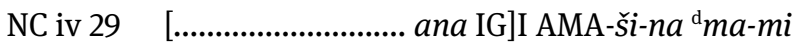

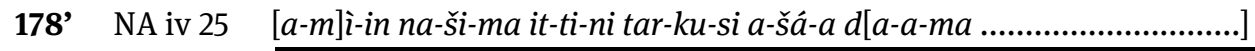

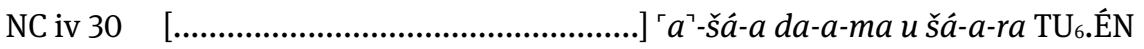

179’ NA iv 26 [KA.IN]IM.MA [...............................]

NC iv 31 .] 'IGI’ GIG.GA.' 'A?'KÂM

180’ [dudubi] šīpāti sāmāti šīpāti peșâti ahennâ tațammi ina qablišunu lippa talpap

181’ šīpāti sāmāti ina īnīšu marušti šīpāti peșâti ina īnišu balitti tarakkasma ina'eš
${ }^{180}$ '[Its medical application]: You spin separately red and white wool, make a wad from their middle (parts), ${ }^{181}$ 'bind the [red wool] on his sick eye and the white wool on his healthy eye, and he will get better.

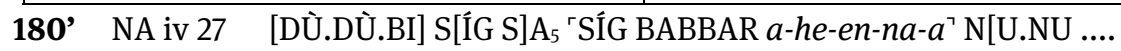

NC iv 32 $a-h e-e] n-{ }^{-} n a-a$ NU.NU ina MURUB 4 -šú-nu? lip-pa tál-pap

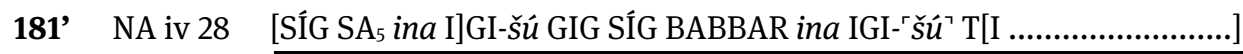

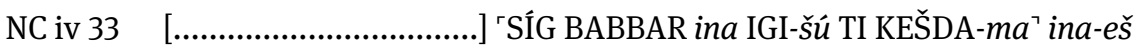

Overview of IGI 1 Manuscripts and Parallels: AA=BAM 20; AB=BAM 159; AC=BAM 19; AD=BAM 22; AE=BAM 18; AmA=BAM 165; AX=KAL 7/12; bA=BM 41293+; EA=EMAR 6/4; HA=STT 279; NA=BAM 510; NB=BAM 513; NC=BAM 514; ND=BAM 515; NE=BAM 516; NJ=AMT 12/5; NQ=BAM 521; NU=AMT 14/3; sA=IRAQ 65; UA=AuOrS 23/27; uA=SpTU 50; UB=Ugaritica 5; UC=AuOrS 23/14; xA=CM 37 
182’ [šiptu še l]á še lá hu-tu-ul hu-tu-ul igi lal hu-tu-ul min igi lal-bi hu-tu-ul min

183’ [šatti] pāna magir šatti pāna magir ițâb magir šanîš tê šipti

184’ ka'inimma šumma amēlu ìnāšu dalhā
182' [Incantation: the winnowed grain], the winnowed grain! It is afflicted, the diminished eye (vision) is afflicted, it is afflicted, ditto, that diminished eye is afflicted, ditto! ${ }^{183}$ 'In the spring it was favourable, in the spring it was favourable! It will be good (and) favourable, secondly (it will be good and favourable)! Incantation spell.

${ }^{184}$ Invocation: if the man's eyes are troubled.

182’ NA iv 29 [ÉN še l]á še lá hu-tu-ul hu-tu-ul igi lal 'hu`-t[u-ul ...........................]

NC iv 34 [......... hu-tu]-`ul hu-tu-ul igi lal hu-tu-ul min igi lal-bi hu-tu-ul min ${ }^{\ulcorner}$

183’ NA iv $30 \quad[\check{s} a-a t-t i p] a-n a$ ŠE.GA ša-at-ti pa-na Š[E.GA .....................................]

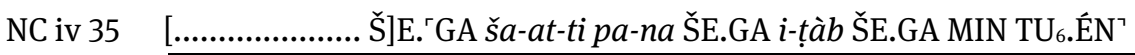

184' NA iv 31 [KA.INI]M.MA DIŠ N[A ................] L[Ü.LÙ]

NC iv $36 \quad[\ldots . . . . . . . . .].]^{~ ' D I S ̌ ~ N A ~ I G I . M I N-s ̌ u ́ ~ L U ̈ . L U ̀ ? ~}$

(ND) iv 45’ [DIŠ NA] IGI.MIN-šú LÙ.LÙ LÀL BABBAR ina İ [..................................]

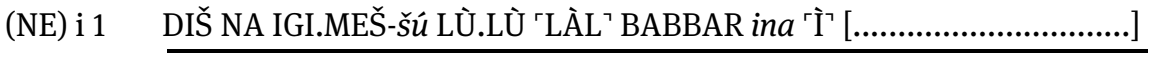

(xA) r.9 [DI]Š NA IGI.MIN-šú LÙ.LÙ-`ha? LÀL İ.NUN.NA İ.'SAG ${ }^{\urcorner}$1-niš HE.HE ina $\mathrm{GE}_{6}$ IGI.MIN-šú DIR[I]

(uA) r.8f. DIŠ NA IGI.MEŠ-šú LÙ.LÙ-ha LÀL İ.NUN.NA İ.SAG gloss: hal-șa 1-niš x ERASURE? KÚM.KÚM-am ta-šá-hal ana IGI.MIN-šú ŠUB

185' [dudubi] birki immeri teleqqe [...] u? țurra tațammi ana šinī̌u teșșip ... [...]

186’ sebet [u] sebet kișrī takașșar ēma takașșaru šipta tamannu ina nakkaptī̌su tarakkasma ina'ěs
${ }^{185}$ 'Its medical application]: take sheep testicle (lit. 'knee') [...], and you spin (and) twine a two-ply cord ... [...], ${ }^{186}$ you shall tie seven [and] seven [knots]. Whenever you make a knot, you shall recite the incantation (see above), you

Overview of IGI 1 Manuscripts and Parallels: AA=BAM 20; AB=BAM 159; AC=BAM 19; AD=BAM 22; AE=BAM 18; AmA=BAM 165; AX=KAL 7/12; bA=BM 41293+; EA=EMAR 6/4; HA=STT 279; NA=BAM 510; NB=BAM 513; NC=BAM 514; ND=BAM 515; NE=BAM 516; NJ=AMT 12/5; NQ=BAM 521; NU=AMT 14/3; sA=IRAQ 65; UA=AuOrS 23/27; uA=SpTU 50; UB=Ugaritica 5; UC=AuOrS 23/14; xA=CM 37 
bind it on his temple, (and) he will get better.

185’ NA iv 32 D[Ü.DÙ.BI $b]$ ir- $^{\ulcorner} k i$ UDU TI- $q e^{\urcorner}[\mathrm{x} \mathrm{x}$ ] $u$ DUR NU.NU ana 2-šú te-eș-ṣi-ip $\mathrm{x}[\mathrm{x} \mathrm{x}]$

NC iv 37f. [...................................] x x x (x) `u? DUR NU.NU ana 2-šú te-eṣ-și-ip / $\left[\begin{array}{lll}\mathrm{x} & \mathrm{x} & \mathrm{x}\end{array}\right]$

186' NA iv $33 \quad 7[u 7$ É]N? ŠID-nu ina SAG.KI-šú KEŠDA- $m[a$.......]

NC iv 38 [..... $]^{\ulcorner 7}$ KA.KEŠDA KEŠDA $e$-ma KEŠDA ÉN ŠID-nu ina SAG.KI-šú KEŠDA-ma ina-eš

187’ šiptu [attina] ìnā pursimìt dāmi šuharratu ammīni taššâni hāma

188' handabillu šuršurra alapâ ša nāri ina sūqāti kirbāna

189' ina tukqinnāte huṣāba ammīni taššâni zunnāni kīma kakkabi

190’ mitaqqutāni kīma nabli lām ikšudūkināši șurru u naglabu

191’ ša Gula šipat lā pațāri šipat Asalluhi Marduk šipat Ningirimma bēlet šipti

192’ u Gula bēlet asûti iddīma anāku ašši tê šipti

193’ ka'inimma hāmū huṣāba u mimma ša ìnī šūlî
187'Incantation: O [you] eyes are porous blood vessels! Why have you picked up chaff, ${ }^{188}$ 'the handabillu-pebbles?, šuršurru-fibre?, the alapu-algae of the river, the clods from the streets (and) ${ }^{189}$ 'twigs from the refuse heaps? Why have you carried (them) along? Rain down like (a shooting) star, ${ }^{190}$ 'drop down like a meteor (lit. flame), before ${ }^{191}$ 'Gula's ${ }^{190}$ 'flint-razor and scalpel manage to reach you (i.e. the eyes). ${ }^{191} \mathrm{An}$ irreversible incantation, the incantation of Asalluhi and Marduk, the incantation of Ningirimma, mistress of incantations, ${ }^{192}$ 'and Gula, mistress of the medical skill; she casts (the spell) and I (the $a$ sûhealer) carry (it) out. Incantation spell. ${ }^{193}$ 'Invocation for removing the chaff, splinter and anything (else) from the eyes.

187’ NA iv 34 ÉN $a[t-t i-n a ~ . . . . . . . . ~ p u] r-{ }^{\ulcorner}$si-mi-it ${ }^{\urcorner}$MÚD šu-har-ra-tú am-mi-ni ta-aš-ša-ni $h a-{ }^{r} m a{ }^{\top}$

Overview of IGI 1 Manuscripts and Parallels: AA=BAM 20; AB=BAM 159; AC=BAM 19; AD=BAM 22; AE=BAM 18; AmA=BAM 165; AX=KAL 7/12; bA=BM 41293+; EA=EMAR 6/4; HA=STT 279; NA=BAM 510; NB=BAM 513; NC=BAM 514; ND=BAM 515; NE=BAM 516; NJ=AMT 12/5; NQ=BAM 521; NU=AMT 14/3; sA=IRAQ 65; UA=AuOrS 23/27; uA=SpTU 50; UB=Ugaritica 5; UC=AuOrS 23/14; $\mathbf{x A = C M ~} 37$ 
NC iv 39' [.................. 'i-na pur-si-mi-it MÚD šu-har-ra-tú am-mi-ni ta-aš-šâ-ni ha-ma

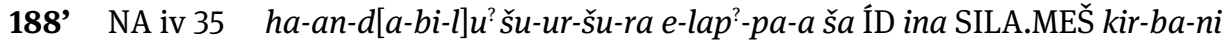
NC iv 40' [.............. ' `̌̌u-úr-šu-ri-im a-lap-pa-na?-a ša ÍD ina SILA.MEŠ kir-ba-ni

189’ NA iv 36 ina tub-kí[n-na]-` $a^{\urcorner}$-te hu-șa-a-ba am-mi-ni taš-ša-ni zu-un-na-ni GIN ${ }_{7}$ MUL

NC iv 41’ [.........................t-t]e rhu-șa-a-ba am-mi-ni taš-ša-ni zu-un-na-ni GIN MUL

190' NA iv 37 mi-ta-q[u-ta]-ni GIN nab-li la-am ik-šu-du-ki-na-ši șur-ru nag-la-bu NC iv 42’ [mi-t]a-'qu-ta-ni GIN ${ }_{7}$ nab-li la-am ik-šu-du-ki-na-šs șur-ru u nag-la-bu ${ }^{\top}$

191’ NA iv 38 šâ ${ }^{\mathrm{d}}$ gu-la É[N N]U DU ÉN $^{\mathrm{d}}$ asal-lú-hi ${ }^{\mathrm{d}} \mathrm{AMAR}$.UTU ÉN ${ }^{\mathrm{d}}$ nin-gi-rim-ma EN ÉN

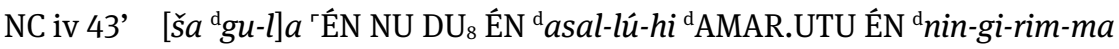
EN ÉN?

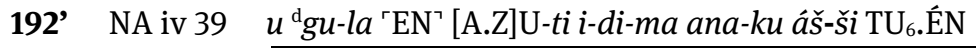

NC iv 44'

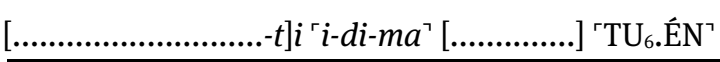

193’ NA iv 40 KA.INIM.MA `ha- $a^{\urcorner}$-mu hu-șa-ba u mìm-ma ša IGI.MIN šu-li-i

NC iv 45’ [KA.INIM.M]A `ha-a-mu hu-șa-ba u mìm-ma IGI.MIN šu-li-i $\urcorner^{\prime}$

194’ šiptu ina šurrî lām bašāmu alallû urda ana māti

195' ittû šer'a ūlid šerhu habburra

196' habburra kanna kannu kișra kișru šubulta šubultu

197’ mer'a Šamaš eșșid Sîn upahhar Šamaš ina eșēdišu Sîn ina puhhurǐšu
${ }^{194}$ 'Incantation. In the beginning before creation, the shaduf descended (from heaven) to earth, ${ }^{195}$ 'the seeder-plough gave birth to the furrow, the furrow to the sprout, ${ }^{196}$ 'the sprout to the rootstock, the root-stock to the bud, the bud to the head-of-grain, (and) the head-ofgrain to ${ }^{197}$ 'the merhu-kernel. The Sungod was harvesting, the Moon-god was reaping. While the Sun-god was

Overview of IGI 1 Manuscripts and Parallels: AA=BAM 20; AB=BAM 159; AC=BAM 19; AD=BAM 22; AE=BAM 18; AmA=BAM 165; AX=KAL 7/12; bA=BM 41293+; EA=EMAR 6/4; HA=STT 279; NA=BAM 510; NB=BAM 513; NC=BAM 514; ND=BAM 515; NE=BAM 516; NJ=AMT 12/5; NQ=BAM 521; NU=AMT 14/3; sA=IRAQ 65; UA=AuOrS 23/27; uA=SpTU 50; UB=Ugaritica 5; UC=AuOrS 23/14; xA=CM 37 
198' ana ìn eṭli merhu īrub Šamaš u Sîn išizzānimma merhu lilâ [tê šipti]

199’ ka'inimma merhu ša libbi īnī šūlî harvesting (and) the Moon-god was reaping, ${ }^{198}$ 'the merhu-kernel entered into the eye of the lad. O Sun-god and Moon-god stand by me, and let the merhu-kernel take off! [Incantation spell.] ${ }^{199}$ 'Invocation for removing the merhu from out of the eyes.

194’ NA iv 41 ÉN ina šur-ri-i la- ${ }^{\ulcorner} a m^{\urcorner}$ba-ša-mu a-la-lu ur-da ana ma-t[i]

$\mathrm{NC}$ iv 46' [........................ba-ša-m]u [a-l]a-l[u] 'ur-da ana $m a^{\urcorner}-t[i]$

195’ NA iv 42 it-tu-ú še-er-a Ù.TU še-er-hu hab-bur-r[a]

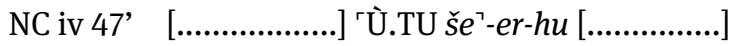

196' NA iv 43 hab-bur-ra ka-an-na 'ka'-an-nu ki-iṣ-ra ki-iṣ-ru šu-bu-ul-ta šu-bu[l-tu] NC iv 48, [............................ ka-an]- ${ }^{n} n u$ ki-iṣ-ra ki-iṣ-ru šu-bu'-[ul-ta ..............]

197’ NA iv 44 mé-er- ${ }^{\mathrm{d}} \mathrm{UTU}$ e-și- $i d^{\mathrm{d}} 30$ ú-pa-har ${ }^{\mathrm{d}} \mathrm{UTU}$ ina e-șe-di-šú ${ }^{\mathrm{d}} 30$ ina pu-h[u$r i$-šú]

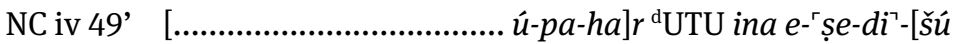
.......]

198’ NA iv 45 ana IGI GURUŠ me-er-hu $\mathrm{KU}_{4}{ }^{\ulcorner} u b^{\urcorner}{ }^{\mathrm{d}} \mathrm{UTU} u$ d30 $i$-ši-za-nim-ma mé-er-hu li-la- $\left.{ }^{\circ} a\right\urcorner\left[\mathrm{TU}_{6} . \mathrm{E} \mathrm{N}\right]$

NC iv 50' .] $\left.\ulcorner i-s ̌ i\urcorner-[z a-n i] m-{ }^{\ulcorner} m a\right\urcorner m e ́-e[r-h u$ ..]

199’ NA iv 46 KA.INIM.MA mé-er-hu 'ša ŠÀ IGI.MIN š[u-li-i]

NC iv 51’ [KA.INIM.MA me-er-hu ša] 'ŠÀ IGI.MIN šu’-[li-i]

\section{Catchline of Tablet Two}

200’ šumma amēlu īnāšu murdinnī malâ sahlê qēm labti ina šikari tarabbak tașammid UD.3.KAM ìnišu [lā tapațtar]

\section{Catchline of Tablet Two}

${ }^{200}$ 'If a man's eyes are full of murdinnubrambles: you shall make an infusion from sahlû-plant (and) flour of roasted grain in beer, (and) bandage (his eyes).

Overview of IGI 1 Manuscripts and Parallels: AA=BAM 20; AB=BAM 159; AC=BAM 19; AD=BAM 22; AE=BAM 18; AmA=BAM 165; AX=KAL 7/12; bA=BM 41293+; EA=EMAR 6/4; HA=STT 279; NA=BAM 510; NB=BAM 513; NC=BAM 514; ND=BAM 515; NE=BAM 516; NJ=AMT 12/5; $\mathbf{N Q = B A M ~ 5 2 1 ; ~ N U = A M T ~ 1 4 / 3 ; ~ S A = I R A Q ~ 6 5 ; ~ U A = A u O r S ~ 2 3 / 2 7 ; ~} \mathbf{u A}=$ SpTU 50; UB=Ugaritica 5; UC=AuOrS 23/14; $\mathbf{x A = C M ~} 37$ 


\begin{tabular}{|l|l|}
\hline & $\begin{array}{l}\text { For three days [you do not loosen (the } \\
\text { bandage from)] his eyes. }\end{array}$ \\
\hline
\end{tabular}

200’ NA iv 47 DIŠ NA IGI.MIN-šú mur-din-n[i DIRI] sah-lé-e Zİ [................] KA[Š ......

(ND) i $1 \quad[$............................ mur-di]n-ni DIRI sah-lé-e Zİ ŠE.SA.A ina KAŠ tarabak LAL UD.3.KÁM IGI.MIN-š[ú NU DU $]$

\begin{tabular}{|l|l|}
\hline $\begin{array}{l}\text { Name of Tablet One } \\
\text { 201' [ištēn] tuppu šumma [amēlu ìnāšu } \\
\text { marșā] }\end{array}$ & $\begin{array}{l}\text { Name of Tablet One } \\
\text { 201'[First] Tablet [of the (Treatise)] 'If } \\
\text { [man's eyes are sick'.] }\end{array}$ \\
\hline
\end{tabular}

201’ NA iv 48 DU[B.1?.KÁM?'] DI[Š NA? IGI?.MIN'-šú? GIG?]

Overview of IGI 1 Manuscripts and Parallels: AA=BAM 20; AB=BAM 159; AC=BAM 19; AD=BAM 22; AE=BAM 18; AmA=BAM 165; AX=KAL 7/12; bA=BM 41293+; EA=EMAR 6/4; HA=STT 279; NA=BAM 510; NB=BAM 513; NC=BAM 514; ND=BAM 515; NE=BAM 516; NJ=AMT 12/5; NQ=BAM 521; NU=AMT 14/3; SA=IRAQ 65; UA=AuOrS 23/27; uA=SpTU 50; UB=Ugaritica 5; UC=AuOrS 23/14; xA=CM 37 